\title{
An algebraic method for moving source localization using TDOA, FDOA, and differential Doppler rate measurements with receiver location errors
}

\author{
Zhixin Liu, Dexiu Hu* (D), Yongsheng Zhao and Yongjun Zhao
}

\begin{abstract}
To weaken the effect of receiver location error on localization accuracy and make the localization model closer to the practical scenario, this paper considers the receiver location errors, usually neglected in prior studies into the measurement model, and proposes an algebraic method for locating a moving source using time difference of arrival (TDOA), frequency difference of arrival (FDOA), and differential Doppler rate measurements. The proposed method is based on the pseudo-linear set of equations and two-step weighted least square estimator. Only noise values of receiver locations and three types of positioning measurements are available for processing. In addition, a new Cramér-Rao lower bound (CRLB) combining TDOA, FDOA, and differential Doppler rate in the presence of receiver location errors is also derived in this paper. Theoretical analysis and simulation results both indicate that the proposed method can attain CRLB at a moderate noise level, avoid the rank deficiency problem efficiently, and achieve a significant improvement over the existing methods.
\end{abstract}

Keywords: Moving source localization, Time difference of arrival, Frequency difference of arrival, Differential Doppler rate, Receiver location errors

\section{Introduction}

Emitter localization based on multiple passive moving receivers has been receiving growing attention for many years due to its important military and civilian application values [1-4]. Passive localization usually includes two steps: firstly, several receivers intercept the signal emitted by a source and measure the proper positioning measurements such as received signal strength (RSS), angle of arrival (AOA), time of arrival (TOA), time difference of arrival (TDOA), and frequency difference of arrival (FDOA), and then use them to obtain the source location [5-14]. The estimation accuracy of RSS is sensitive to the channel environment. If the practical environment exists strong scatters, the signal strength is difficult to estimate which influences on localization accuracy [6]. The TOA-and-AOA-based localization system can provide an accurate source position estimate, at

\footnotetext{
* Correspondence: paper_hdx@126.com

National Digital Switching System Engineering and Technological Research Center (NDSC), Science Road 62, Zhengzhou, People's Republic of China
}

the cost of requiring time synchronization between receivers and source, and complicated antenna hardware $[7,8]$. For non-cooperative source localization problem, the TDOA can be utilized to improve localization accuracy. If there is relative motion between the source and receivers, the FDOA can be combined with the TDOA, which can significantly improve the source location accuracy and estimate the position and velocity of the source simultaneously [10-14].

As for high-dimensional and nonlinear characteristic of TDOA-and-FDOA-based localization problem, many efficient methods have been proposed. Foy put forward a linearization method by using Taylor expansion [11], but a proper initial guess is necessary, or its convergence cannot be guaranteed. To avoid it, the algebraic methods have always been compelling to researchers due to their independence of initial estimate and computational efficiency. Ho and $\mathrm{Xu}$ proposed the well-known two-step weighted least square (TSWLS) method [10] that gives an algebraic solution for moving source localization 
without initial guess. However, this method has poor localization accuracy when a source is near to any coordinate axis of the reference receiver and needs several extra mathematical operations before determining the source location in the second step. After that, the semi-definite relaxation methods are applied in a localization system [12, 13]. Essentially, these methods are also an iteration-based method and always suffer from a high computational complexity. Recently, an improved TSWLS method was presented in [14], which outperforms the original TSWLS method.

As we know, the source localization accuracy depends much on the TDOA and FDOA estimation accuracy. Hence, to improve the performance of radar detection and parameter estimation, some studies aim at the long-time coherent integration $[15,16]$. Nevertheless, extending the observation time would improve FDOA estimation accuracy, which has contributed to source localization [17], but it will cause serious relative Doppler companding problem $[17,18]$. To overcome it, several efforts considered the differential Doppler rate in the traditional signal model and proposed the joint estimation method, from which TDOA, FDOA, and differential Doppler rate can be obtained simultaneously [1820]. It should be noted that this measurement can be obtained jointly with TDOA and FDOA requiring no additional data collection device, which means using the same received data with TDOA and FDOA estimation $[21,22]$. Moreover, it is closely associated with the source position and velocity. Hence, in the localization system, ignoring the differential Doppler rate measurement may not be acceptable for many scenarios with high maneuvering or long observation time [20] and is utilized to determine the source location. Quite recently, $\mathrm{Hu}$ et al. proposed a gradient method using TDOA, FDOA, and differential Doppler rate measurements [17], but the iteration process of the second step could cause local convergence or even divergence at low signal-to-noise ratio (SNR) conditions.

Generally, the above localization methods are all based on the ideal assumption that the receiver locations are known exactly, which is not realistic for practical scenario $[23,24]$. The receiver locations are inevitably contaminated by errors to some extent and are often non-negligible, especially in practical environments. Ho et al. proved that the source localization accuracy is very sensitive to the accuracy of receiver locations and developed a solution with random location errors [25], but neglects the differential Doppler rate measurement in solving step. Moreover, the second step of this method has poor robustness due to its rank deficiency problem, which is analyzed in the simulation part of this paper.

Motivated by the above facts, this paper takes receiver location errors into consideration in the measurement model and proposes an algebraic method for moving source localization using TDOA, FDOA, and differential Doppler rate measurements. Inspired by the idea of the TSWLS method, the measurement equations are linearized by introducing three additional parameters and a rough estimate is obtained by using weighted least square (WLS) estimator. Then, a final algebraic solution is presented by utilizing the relationship between the additional parameters and the source location through another WLS. The contributions of this paper include the following aspects.

1. The receiver location errors are considered to offset the weakness of the existing methods, which can produce a more reliable estimation result of source location.

2. The second step of the proposed method gives a final algebraic solution, while the existing methods need extra mathematics operations, such as multiple iterations in [17] and square root operations in [25].

3. The proposed method can efficiently avoid rank deficiency problem and is more robust than the TSWLS method in some special localization scenarios where source moves near to any coordinate axis of the reference receiver.

4. The proposed method outperforms the existing methods with respect to localization accuracy as SNR and receiver location error change. Simulation results can support the theoretical analysis and the above contributions.

The rest of paper is organized as follows. Section 2 introduces the method and experimentally used in this paper. Section 3 formulates the problem of source localization using TDOA, FDOA, and differential Doppler rate measurements with receiver location errors. Section 4 gives a detailed derivation of the proposed method. Section 5 derives a new Cramér-Rao lower bound (CRLB) and gives a comparison between the estimation accuracy of the proposed method and CRLB. Section 6 provides numerous simulations to support our theoretical study and evaluate the localization accuracy of the proposed method. Section 8 concludes this paper. Some important derivations are given in Appendix 1 and 2.

This paper contains a number of symbols. Following the convention, we represent the matrixes and vectors as bold italic case letters. The operations $[.]^{T}$ and $[.]^{-1}$ denote the matrix transpose and inverse. $E[$. ], $\|$. $\|$, and | . | represent the mathematical expectation, 2-norm, and determinant, respectively. The superscript $(\cdot)^{o}$ and ( . ) are used to distinguish between the true value and noisy value. $\boldsymbol{\alpha}(i: j)$ stands for a column sub-vector including the $i^{\text {th }}$ to the $j^{\text {th }}$ element of the vector $\boldsymbol{\alpha}$. $\mathbf{V}_{i \times i}$ is a $i$ 
square matrix with 1 in the diagonal and 0.5 in all other elements. $\mathbf{0}_{i \times j}$ denotes a $i \times j$ vector of zeros. $\mathbf{O}_{i \times j}$ and $\mathbf{I}_{i \times j}$ represent a $i \times j$ zero matrix and identity matrix.

\section{Methods/experimental}

Despite the rapid development of the localization method, it is still quite a challenge owing to the increasing demand for high localization accuracy. This paper considers the receiver location errors in the traditional measurement model and gives an algebraic solution using TDOA, FDOA, and differential Doppler rate measurements, aiming to produce a robust and accurate source location. The proposed method is designed based on the idea of the TSWLS method. We transform the localization equations into a pseudo-linear set of the equation by introducing three additional parameters, and a rough estimate is obtained in a WLS sense. Then, a final algebraic solution is given by using the additional parameters through another WLS. To evaluate the performance of the proposed method, we derive a new CRLB on position and velocity estimation with receiver location errors and also analysis of the relationship between the covariance of the method and the CRLB.

A set of Monte Carlo simulations is conducted to examine the behavior of the proposed method. The root mean square errors (RMSEs) are used to evaluate the estimation accuracy for source position and velocity, which is acquired from 5000 independent trials. In order to prove the necessity of considering the receiver location errors during the design of methods, we compare the CRLB derived in this paper with the CRLB in [17] as SNR and receiver location errors change. Then, to exhibit the superiority of the proposed method in robustness and performance, we compare our method with Hu's method, TSWLS method, as well as the three types of CRLBs in three-dimensional (3-D) scenario. Besides, all the experiments are performed in two noise conditions including measurement noise and receiver location error noise.

This paper does not contain any studies with human participants or animals performed by any of the authors.

\section{Problem formulation}

Considering a 3-D scenario including $M$ passive moving receivers at position $\mathbf{s}_{i}=\left[x_{i}, y_{i}, z_{i}\right]^{T}$ with velocity $\dot{\mathbf{s}}_{i}$ $=\left[\dot{x}_{i}, \dot{y}_{i}, \dot{z}_{i}\right]^{T}$ for $i=1,2, \ldots, M$, we are interested in identifying the unknown emitting source position $\boldsymbol{u}=[x, y$, $z]^{T}$ and velocity $\dot{\boldsymbol{u}}=[\dot{x}, \dot{y}, \dot{z}]^{T}$ using TDOA, FDOA, and differential Doppler rate measurements. Stacking the true value $\boldsymbol{s}_{i}^{o}$ and $\dot{\boldsymbol{s}}_{i}^{o}$ together for $i=1,2, \ldots, M$ leads to a $6 M \times 1$ column vector as follows:

$$
\boldsymbol{\beta}^{o}=\left[\boldsymbol{s}^{o T}, \dot{\boldsymbol{s}}^{o T}\right]^{T}
$$

with $\boldsymbol{s}^{o}=\left[\boldsymbol{s}_{1}^{o T}, \boldsymbol{s}_{2}^{o T}, \ldots, \boldsymbol{s}_{M}^{o T}\right]^{T}$ and $\dot{\boldsymbol{s}}^{o}=\left[\dot{\boldsymbol{s}}_{1}^{o T}, \dot{\boldsymbol{s}}_{2}^{o T}, \ldots, \dot{\boldsymbol{s}}_{M}^{o T}\right]^{T}$. Note that in practice, the true value of the receiver position $\boldsymbol{s}^{o}$ and velocity $\dot{\boldsymbol{s}}^{o}$ are unknown to an estimator, and only the noisy value $\boldsymbol{s}$ and $\dot{s}$ are available for deriving the method in this paper. The vector forms the noise value and error which are respectively denoted by

$$
\begin{aligned}
& \boldsymbol{\beta}=\left[\boldsymbol{s}^{T}, \dot{\boldsymbol{s}}^{T}\right]^{T} \\
& \Delta \boldsymbol{\beta}=\boldsymbol{\beta}-\boldsymbol{\beta}^{o}=\left[\Delta \boldsymbol{s}^{T}, \Delta \dot{\boldsymbol{s}}^{T}\right]^{T}
\end{aligned}
$$

where $\Delta \boldsymbol{s}=\left[\Delta \boldsymbol{s}_{1}^{T}, \Delta \boldsymbol{s}_{2}^{T}, \ldots, \Delta \boldsymbol{s}_{M}^{T}\right]^{T}$ with $\Delta \boldsymbol{s}_{i}=\boldsymbol{s}_{i}-\boldsymbol{s}_{i}^{o}$ for $i=$ $1,2, \ldots, M, \Delta \dot{\boldsymbol{s}}=\left[\Delta \dot{\boldsymbol{s}}_{1}^{T}, \Delta \dot{\boldsymbol{s}}_{2}^{T}, \ldots, \Delta \dot{\boldsymbol{s}}_{M}^{T}\right]^{T}$ with $\Delta \dot{\boldsymbol{s}}_{i}=\dot{\boldsymbol{s}}_{i}-\dot{\boldsymbol{s}}_{i}^{o}$ for $i=1,2, \ldots, M$. $\Delta \boldsymbol{\beta}$ is assumed to follow zero mean Gaussian distribution with covariance matrix

$$
E\left[\Delta \boldsymbol{\beta} \Delta \boldsymbol{\beta}^{T}\right]=\boldsymbol{Q}_{\boldsymbol{\beta}}
$$

Based on the above definitions, the true range and range rate between the source and the $i^{\text {th }}$ receiver are

$$
\begin{aligned}
r_{i}^{o} & =\left\|\boldsymbol{u}^{o}-\boldsymbol{s}_{i}^{o}\right\| \\
\dot{r}_{i}^{o} & =\frac{\left(\boldsymbol{u}^{o}-\boldsymbol{s}_{i}^{o}\right)^{T}\left(\dot{\boldsymbol{u}}^{o}-\dot{\boldsymbol{s}}_{i}^{o}\right)}{r_{i}^{o}}
\end{aligned}
$$

where $\mathbf{u}^{o}$ and $\dot{\mathbf{u}}^{o}$ stand for the true value of the source location. The second time derivative of the range gives the true acceleration between the source and the $i^{\text {th }}$ receiver

$$
\ddot{r}_{i}^{o}=\frac{\left(\dot{\boldsymbol{u}}^{o}-\dot{\boldsymbol{s}}_{i}^{o}\right)^{T}\left(\dot{\boldsymbol{u}}^{o}-\dot{\boldsymbol{s}}_{i}^{o}\right)-\dot{r}_{i}^{o 2}}{r_{i}^{o}}
$$

It should be explained that from the standpoint of efficiency in the localization system, we assume that the velocity of sensors is constant or nearly constant during the observation window time. Therefore, $\ddot{\mathbf{s}}_{i}=\mathbf{0}_{3 \times 1}$ for $i=1$, $2, \ldots, M$. But the way, we would like to say that if there exist a distinct acceleration of sensors, the proposed method can also accomplish the source localization and the localization performance would be better because additional information can be used and the differential Doppler rate would be more significantly $[10,14,17]$. Without loss of generality, the first receiver is chosen as the reference receiver to derive the proposed method in the following paper. The true TDOA, FDOA, and differential Doppler rate between the $i^{\text {th }}$ receiver and the reference receiver are respectively given by

$$
\begin{aligned}
& r_{i 1}^{o}=c \tau_{i 1}^{o}=r_{i}^{o}-r_{1}^{o} \\
& \dot{r}_{i 1}^{o}=\lambda f_{i 1}^{o}=\dot{r}_{i}^{o}-\dot{r}_{1}^{o} \\
& \ddot{r}_{i 1}^{o}=\lambda \dot{f}_{i 1}^{o}=\ddot{r}_{i}^{o}-\ddot{r}_{1}^{o}
\end{aligned}
$$

where $i=2,3, \ldots, M . c$ is the signal propagation speed, and $\lambda$ is the wavelength of the received signal. $\tau_{i 1}^{o}, f_{i 1}^{o}$, and $\dot{f}_{i 1}^{o}$ are respectively the true TDOA, FDOA, and dif- 
ferential Doppler rate. For simplicity, we use $r_{i 1}^{o}, \dot{r}_{i 1}^{o}$, and $\ddot{r}_{i 1}^{o}$ to derive the proposed method in this paper. Consider the measurement noises in practice, and the TDOA, FDOA, and differential Doppler rate measurements can be expressed as

$$
\begin{aligned}
& r_{i 1}=r_{i 1}^{o}+\Delta r_{i 1} \\
& \dot{r}_{i 1}=\dot{r}_{i 1}^{o}+\Delta \dot{r}_{i 1} \\
& \ddot{r}_{i 1}=\ddot{r}_{i 1}^{o}+\Delta \ddot{r}_{i 1}
\end{aligned}
$$

where $\Delta r_{i 1}, \Delta \dot{r}_{i 1}$, and $\Delta \ddot{r}_{i 1}$ denote the TDOA, FDOA, and differential Doppler rate measurement errors, respectively, from $i$ th receivers. Stacking them together for $i=$ $2,3, \ldots, M$ yields the vector form

$$
\begin{aligned}
& \mathbf{r}=\mathbf{r}^{o}+\Delta \mathbf{r} \\
& \dot{\mathbf{r}}=\dot{\mathbf{r}}^{o}+\Delta \mathbf{r} \\
& \ddot{\mathbf{r}}=\ddot{\mathbf{r}}^{o}+\Delta \ddot{\mathbf{r}}
\end{aligned}
$$

where

$$
\begin{aligned}
& \mathbf{r}=\left[r_{21}, r_{31}, \ldots, r_{M 1}\right]^{T}, \mathbf{r}^{o}=\left[r_{21}^{o}, r_{31}^{o}, \ldots, r_{M 1}^{o}\right]^{T}, \Delta \mathbf{r}=\left[\Delta r_{21}, \Delta r_{31}, \ldots, \Delta r_{M 1}\right]^{T} \\
& \dot{\mathbf{r}}=\left[\dot{r}_{21}, \dot{r}_{31}, \ldots, \dot{r}_{M 1}\right]^{T}, \dot{\mathbf{r}}^{o}=\left[\dot{r}_{21}^{o}, \dot{r}_{31}^{o}, \ldots, \dot{r}_{M 1}^{o}\right]^{T}, \Delta \mathbf{r}=\left[\Delta \dot{r}_{21}, \Delta \dot{r}_{31}, \ldots, \Delta \dot{r}_{M 1}\right]^{T} \\
& \ddot{\mathbf{r}}=\left[\ddot{r}_{21}, \ddot{r}_{31}, \ldots, \ddot{r}_{M 1}\right]^{T}, \ddot{\mathbf{r}}^{o}=\left[\ddot{r}_{21}^{o}, \ddot{r}_{31}^{o}, \ldots, \ddot{r}_{M 1}^{o}\right]^{T}, \Delta \ddot{\mathbf{r}}=\left[\Delta \ddot{r}_{21}, \Delta \ddot{r}_{31}, \ldots, \Delta \ddot{r}_{M 1}\right]^{T}
\end{aligned}
$$

Define $\quad \Delta \mathbf{\alpha}=\left[\Delta \mathbf{r}^{T}, \Delta \dot{\mathbf{r}}^{T}, \Delta \ddot{\mathbf{r}}^{T}\right]^{T}$ as the measurement error vector, which follows the zero mean Gaussian distribution with covariance matrix $E\left[\Delta \boldsymbol{\alpha} \Delta \boldsymbol{\alpha}^{T}\right]=\mathbf{Q}_{\boldsymbol{\alpha}}$. Our primary objective is to estimate the source position $\mathbf{u}$ and velocity $\dot{\mathbf{u}}$ as accurately as possible using the noisy measurement vector $\boldsymbol{\alpha}=\left[\mathbf{r}^{T}, \dot{\mathbf{r}}^{T}, \ddot{\mathbf{r}}^{T}\right]^{T}$ with noisy receiver location vector $\beta$.

\section{Localization method}

It can be seen that (8) is nonlinear concerning the unknown source position and velocity, which is not a trivial task to solve these nonlinear measurement equations directly. The work in [17] uses the iteration-based method to solve these, which exists the convergence problem. Hence, this paper is inspired by the idea of WLS and introduces two steps to obtain the source location including a rough estimation step and a refined estimation step. The first step solution is introduced in the following:

\subsection{First WLS step}

From the source localization model (6), rewrite the TDOA equations as $r_{i 1}^{o}+r_{1}^{o}=r_{i}^{o}$, square both sides, substitute $r_{i}^{o 2}=\left(\boldsymbol{u}^{o}-\boldsymbol{s}_{i}^{o}\right)^{T}\left(\boldsymbol{u}^{o}-\boldsymbol{s}_{i}^{o}\right)$ for $r_{i}^{o 2}$ and $r_{1}^{o 2}$, and a set of TDOA equations can be expressed as

$$
r_{i 1}^{o 2}+2 r_{i 1}^{o} r_{1}^{o}=\left(\mathbf{s}_{i}^{o T} \mathbf{s}_{i}^{o}-\mathbf{s}_{1}^{o T} \mathbf{s}_{1}^{o}\right)-2\left(\mathbf{s}_{i}^{o}-\mathbf{s}_{1}^{o}\right)^{T} \mathbf{u}^{o}
$$

Then, take the time derivative of (10), and the FDOA equation is changed as

$$
\dot{r}_{i 1}^{o} r_{1}^{o}+r_{i 1}^{o} \dot{r}_{1}^{o}=\left(\dot{\mathbf{s}}_{i}^{o T} \mathbf{s}_{i}^{o}-\dot{\mathbf{s}}_{1}^{o T} \mathbf{s}_{1}^{o}-\dot{r}_{i 1}^{o} r_{i 1}^{o}\right)-\left(\dot{\mathbf{s}}_{i}^{o}-\dot{\mathbf{s}}_{1}^{o}\right)^{T} \mathbf{u}^{o}-\left(\mathbf{s}_{i}^{o}-\mathbf{s}_{1}^{o}\right)^{T} \dot{\mathbf{u}}^{o}
$$

Taking the second time derivative of (10) gives the new differential Doppler rate equation as

$$
\ddot{r}_{i 1}^{o} r_{1}^{o}+2 \dot{r}_{i 1}^{o} \dot{r}_{1}^{o}+r_{i 1}^{o} \ddot{r}_{1}^{o}=\left(\dot{\mathbf{s}}_{i}^{o T} \dot{\mathbf{s}}_{i}^{o}-\dot{\mathbf{s}}_{1}^{o T} \dot{\mathbf{s}}_{1}^{o}-\ddot{r}_{i 1}^{o} r_{i 1}^{o}-\dot{r}_{i 1}^{o 2}\right)-2\left(\dot{\mathbf{s}}_{i}^{o}-\dot{\mathbf{s}}_{1}^{o}\right)^{T} \dot{\mathbf{u}}^{o}
$$

In the presence of errors including measurement noise in TDOA, FDOA, and differential Doppler rate, and receiver locations, we should replace the true measured values with their noisy terms. Thereby, substitute $r_{i 1}$ $-\Delta r_{i 1}$ and $\boldsymbol{s}_{i}-\Delta \boldsymbol{s}_{i}$ for $r_{i 1}^{o}$ and $\boldsymbol{s}_{i}^{o}$ in (10), ignore the second-order terms, and the TDOA equation can be rewritten as

$$
\begin{aligned}
& -2 r_{i} \Delta r_{i 1}-2\left(\mathbf{u}-\mathbf{s}_{i}\right)^{T} \Delta \mathbf{s}_{i}+2\left(\mathbf{u}-\mathbf{s}_{1}\right)^{T} \Delta \mathbf{s}_{1} \\
& \quad=\left(\mathbf{s}_{i}^{T} \mathbf{s}_{i}-\mathbf{s}_{1}^{T} \mathbf{s}_{1}-r_{i 1}^{2}\right)-2\left(\mathbf{s}_{i}-\mathbf{s}_{1}\right)^{T} \mathbf{u}-2 r_{i 1} r_{1}
\end{aligned}
$$

Replace $r_{i 1}^{o}, \dot{r}_{i 1}^{o}, \boldsymbol{s}_{i}^{o}$, and $\dot{\boldsymbol{s}}_{i}^{o}$ with $r_{i 1}-\Delta r_{i 1}, \dot{r}_{i 1}-\Delta \dot{r}_{i 1}, \boldsymbol{s}_{i}$ $-\Delta \boldsymbol{s}_{i}$, and $\dot{\boldsymbol{s}}_{i}-\Delta \dot{\boldsymbol{s}}_{i}$ in (11), ignore the second-order terms, and the FDOA equation is changed as

$$
\begin{aligned}
& -\dot{r}_{i} \Delta r_{i 1}-r_{i} \Delta \dot{r}_{i 1}-\left(\dot{\mathbf{u}}-\dot{\mathbf{s}}_{i}\right)^{T} \Delta \mathbf{s}_{i}-\left(\mathbf{u}-\mathbf{s}_{i}\right)^{T} \Delta \dot{\mathbf{s}}_{i}+\left(\dot{\mathbf{u}}-\dot{\mathbf{s}}_{1}\right)^{T} \Delta \mathbf{s}_{1}+\left(\mathbf{u}-\mathbf{s}_{1}\right)^{T} \Delta \dot{\mathbf{s}}_{1} \\
& =\left(\dot{\mathbf{s}}_{i}^{T} \mathbf{s}_{i}-\dot{\mathbf{s}}_{1}^{T} \mathbf{s}_{1}-\dot{r}_{i 1} r_{i 1}\right)^{-}-\left(\dot{\mathbf{s}}_{i}-\dot{\mathbf{s}}_{1}\right)^{T} \mathbf{u}-\dot{r}_{i 1} r_{1}-\left(\mathbf{s}_{i}-\mathbf{s}_{1}\right)^{T} \dot{\mathbf{u}}-r_{i 1} \dot{r}_{1}
\end{aligned}
$$

Similarly, replace $r_{i 1}^{o}, \dot{r}_{i 1}^{o}, \ddot{r}_{i 1}^{o}, \boldsymbol{s}_{i}^{o}$, and $\dot{s}_{i}^{o}$ with $r_{i 1}-\Delta r_{i 1}$, $\dot{r}_{i 1}-\Delta \dot{r}_{i 1}, \ddot{r}_{i 1}-\Delta \ddot{r}_{i 1}, s_{i}-\Delta \boldsymbol{s}_{i}$, and $\dot{s}_{i}-\Delta \dot{s}_{i}$ in (11), ignore the second-order terms, and the differential Doppler rate equation can be expressed as

$$
\begin{aligned}
& -\ddot{r}_{i} \Delta r_{i 1}-2 \dot{r}_{i} \Delta \dot{r}_{i 1}-r_{i} \Delta \ddot{r}_{i 1}-2\left(\dot{\mathbf{u}}-\dot{\mathbf{s}}_{i}\right)^{T} \Delta \dot{\mathbf{s}}_{i}+2\left(\dot{\mathbf{u}}-\dot{\mathbf{s}}_{1}\right)^{T} \Delta \dot{\mathbf{s}}_{1} \\
& =\left(\dot{\mathbf{s}}_{i}^{T} \dot{\mathbf{s}}_{i}-\dot{\mathbf{s}}_{1}^{T} \dot{\mathbf{s}}_{1}-\ddot{r}_{i 1} r_{i 1}-\dot{r}_{i 1}^{2}\right)-\ddot{r}_{i 1} r_{1}-2\left(\dot{\mathbf{s}}_{i}-\dot{\mathbf{s}}_{1}\right)^{T} \dot{\mathbf{u}}-2 \dot{r}_{i 1} \dot{r}_{1}-r_{i 1} \ddot{r}_{1}
\end{aligned}
$$

Defining $\boldsymbol{\theta}_{1}^{o}=\left[\mathbf{u}^{o T}, r_{1}^{o}, \dot{\mathbf{u}}^{o T}, \dot{r}_{1}^{o}, \ddot{r}_{1}^{o}\right]^{T}$ and collecting (13) for $i=2,3, \ldots, M$ yields

$$
\begin{aligned}
& {\left[2 \mathbf{B} \mathbf{O}_{(M-1) \times 2(M-1)}\right] \Delta \boldsymbol{\alpha}} \\
& \quad+\left[2 \mathbf{D} \mathbf{O}_{(M-1) \times(3 M)}\right] \Delta \boldsymbol{\beta} \\
& \quad=\mathbf{h}_{1, r}-\mathbf{G}_{1, r} \boldsymbol{\theta}_{1}^{o}
\end{aligned}
$$

where

$$
\begin{aligned}
\mathbf{G}_{1, r} & =2\left[\begin{array}{ccccc}
\left(\mathbf{s}_{2}-\mathbf{s}_{1}\right)^{T} & r_{21} & \mathbf{0}_{1 \times 3} & 0 & 0 \\
\left(\mathbf{s}_{3}-\mathbf{s}_{1}\right)^{T} & r_{31} & \mathbf{0}_{1 \times 3} & 0 & 0 \\
\vdots & \vdots & \vdots & \vdots & \vdots \\
\left(\mathbf{s}_{M}-\mathbf{s}_{1}\right)^{T} & r_{M 1} & \mathbf{0}_{1 \times 3} & 0 & 0
\end{array}\right], \quad \mathbf{h}_{1, r} \\
& =\left[\begin{array}{c}
\mathbf{s}_{2}^{T} \mathbf{s}_{2}-\mathbf{s}_{1}^{T} \mathbf{s}_{1}-r_{21}^{2} \\
\mathbf{s}_{3}^{T} \mathbf{s}_{3}-\mathbf{s}_{1}^{T} \mathbf{s}_{1}-r_{31}^{2} \\
\vdots \\
\mathbf{s}_{M}^{T} \mathbf{s}_{M}-\mathbf{s}_{1}^{T} \mathbf{s}_{1}-r_{M 1}^{2}
\end{array}\right]
\end{aligned}
$$

and 


$$
\begin{aligned}
\mathbf{B} & =-\left[\begin{array}{ccccc}
r_{2} & & & \\
& r_{3} & & \\
& & \ddots & \\
& & & r_{M}
\end{array}\right], \\
\mathbf{D} & =\left[\begin{array}{ccccc}
\left(\mathbf{u}-\mathbf{s}_{1}\right)^{T} & -\left(\mathbf{u}-\mathbf{s}_{2}\right)^{T} & \mathbf{0}_{3 \times 1}^{T} & \cdots & \mathbf{0}_{3 \times 1}^{T} \\
\left(\mathbf{u}-\mathbf{s}_{1}\right)^{T} & \mathbf{0}_{3 \times 1}^{T} & -\left(\mathbf{u}-\mathbf{s}_{3}\right)^{T} & \cdots & \mathbf{0}_{3 \times 1}^{T} \\
\vdots & \vdots & \vdots & \ddots & \vdots \\
\left(\mathbf{u}-\mathbf{s}_{1}\right)^{T} & \mathbf{0}_{3 \times 1}^{T} & \mathbf{0}_{3 \times 1}^{T} & \cdots & -\left(\mathbf{u}-\mathbf{s}_{M}\right)^{T}
\end{array}\right]
\end{aligned}
$$

Stack (14) for $i=2,3, \ldots, M$ together and the matrix equation regarding $\boldsymbol{\theta}_{1}^{o}$ is

$$
\left[\begin{array}{lll}
\dot{\mathbf{B}} & \mathbf{B} & \mathbf{O}_{(M-1) \times(M-1)}
\end{array}\right] \Delta \boldsymbol{\alpha}+\left[\begin{array}{ll}
\dot{\mathbf{D}} & \mathbf{D}
\end{array}\right] \Delta \boldsymbol{\beta}=\mathbf{h}_{1, \dot{r}}-\mathbf{G}_{1, \dot{r}} \boldsymbol{\theta}_{1}^{o}
$$

where

$$
\begin{aligned}
\mathbf{G}_{1, \dot{r}} & =\left[\begin{array}{ccccc}
\left(\dot{\mathbf{s}}_{2}-\dot{\mathbf{s}}_{1}\right)^{T} & \dot{r}_{21} & \left(\mathbf{s}_{2}-\mathbf{s}_{1}\right)^{T} & r_{21} & 0 \\
\left(\dot{\mathbf{s}}_{3}-\dot{\mathbf{s}}_{1}\right)^{T} & \dot{r}_{31} & \left(\mathbf{s}_{3}-\mathbf{s}_{1}\right)^{T} & r_{31} & 0 \\
\vdots & \vdots & \vdots & \vdots & \vdots \\
\left(\dot{\mathbf{s}}_{M}-\dot{\mathbf{s}}_{1}\right)^{T} & \dot{r}_{M 1} & \left(\mathbf{s}_{M}-\mathbf{s}_{1}\right)^{T} & r_{M 1} & 0
\end{array}\right], \\
\mathbf{h}_{1, \dot{r}} & =\left[\begin{array}{c}
\dot{\mathbf{s}}_{2}^{T} \mathbf{s}_{2}-\dot{\mathbf{s}}_{1}^{T} \mathbf{s}_{1}-\dot{r}_{21} r_{21} \\
\dot{\mathbf{s}}_{3}^{T} \mathbf{s}_{3}-\dot{\mathbf{s}}_{1}^{T} \mathbf{s}_{1}-\dot{r}_{31} r_{31} \\
\vdots \\
\dot{\mathbf{s}}_{M}^{T} \mathbf{s}_{M}-\dot{\mathbf{s}}_{1}^{T} \mathbf{s}_{1}-\dot{r}_{M 1} r_{M 1}
\end{array}\right]
\end{aligned}
$$

and

$$
\begin{aligned}
\mathbf{B} & =-\left[\begin{array}{lllll}
\dot{r}_{2} & & & \\
& \dot{r}_{3} & & \\
& & \ddots & \\
& & & \dot{r}_{M}
\end{array}\right], \\
\dot{\mathbf{D}} & =\left[\begin{array}{ccccc}
\left(\dot{\mathbf{u}}-\dot{\mathbf{s}}_{1}\right)^{T} & -\left(\dot{\mathbf{u}}-\dot{\mathbf{s}}_{2}\right)^{T} & \mathbf{0}_{3 \times 1}^{T} & \cdots & \mathbf{0}_{3 \times 1}^{T} \\
\left(\dot{\mathbf{u}}-\dot{\mathbf{s}}_{1}\right)^{T} & \mathbf{0}_{3 \times 1}^{T} & -\left(\dot{\mathbf{u}}-\dot{\mathbf{s}}_{3}\right)^{T} & \cdots & \mathbf{0}_{3 \times 1}^{T} \\
\vdots & \vdots & \vdots & \ddots & \vdots \\
\left(\dot{\mathbf{u}}-\dot{\mathbf{s}}_{1}\right)^{T} & \mathbf{0}_{3 \times 1}^{T} & \mathbf{0}_{3 \times 1}^{T} & \cdots & -\left(\dot{\mathbf{u}}-\dot{\mathbf{s}}_{M}\right)^{T}
\end{array}\right]
\end{aligned}
$$

Gather (15) for $i=2,3, \ldots, M$ together and we have

$$
\left[\begin{array}{lll}
\ddot{\mathbf{B}} & 2 \dot{\mathbf{B}} & \mathbf{B}
\end{array}\right] \Delta \boldsymbol{\alpha}+\left[\mathbf{O}_{(M-1) \times(3 M)} \quad 2 \dot{\mathbf{D}}\right] \Delta \boldsymbol{\beta}=\mathbf{h}_{1, \ddot{r}}-\mathbf{G}_{1, \dot{r}} \boldsymbol{\theta}_{1}^{o}
$$

where

$$
\begin{aligned}
& \mathbf{G}_{1, \ddot{r}}=\left[\begin{array}{ccccc}
\mathbf{0}_{3 \times 1}^{T} & \ddot{r}_{21} & 2\left(\dot{\mathbf{s}}_{2}-\dot{\mathbf{s}}_{1}\right)^{T} & 2 \dot{r}_{21} & r_{21} \\
\mathbf{0}_{3 \times 1}^{T} & \ddot{r}_{31} & 2\left(\dot{\mathbf{s}}_{3}-\dot{\mathbf{s}}_{1}\right)^{T} & 2 \dot{r}_{31} & r_{31} \\
\vdots & \vdots & \vdots & \vdots & \vdots \\
\mathbf{0}_{3 \times 1}^{T} & \ddot{r}_{M 1} & 2\left(\dot{\mathbf{s}}_{M}-\dot{\mathbf{s}}_{1}\right)^{T} & 2 \dot{r}_{M 1} & r_{M 1}
\end{array}\right], \\
& \mathbf{h}_{1, \ddot{r}}=\left[\begin{array}{c}
\dot{\mathbf{s}}_{2}^{T} \dot{\mathbf{s}}_{2}-\dot{\mathbf{s}}_{1}^{T} \dot{\mathbf{s}}_{1}-\ddot{r}_{21} r_{21}-\dot{r}_{11}^{2} \\
\dot{\mathbf{s}}_{3}^{T} \dot{\mathbf{s}}_{3}-\dot{\mathbf{s}}_{1}^{T} \dot{\mathbf{s}}_{1}-\ddot{r}_{31} r_{31}-\dot{r}_{31}^{2} \\
\vdots \\
\dot{\mathbf{s}}_{M}^{T} \dot{\mathbf{s}}_{M}-\dot{\mathbf{s}}_{1}^{T} \dot{\mathbf{s}}_{1}-\ddot{r}_{M 1} r_{M 1}-\dot{r}_{M 1}^{2}
\end{array}\right], \quad \ddot{\mathbf{B}}=-\left[\begin{array}{cccc}
\ddot{r}_{2} & & & \\
& \ddot{r}_{3} & & \\
& & \ddots & \\
& & & \ddot{r}_{M}
\end{array}\right]
\end{aligned}
$$

Now, combine (16), (19) and (22) to form the set of equations extracted from TDOA, FDOA, and differential Doppler rate measurements as

$$
\boldsymbol{\varepsilon}_{1}=\mathbf{B}_{1} \Delta \boldsymbol{\alpha}+\mathbf{D}_{1} \Delta \boldsymbol{\beta}=\mathbf{h}_{1}-\mathbf{G}_{1} \boldsymbol{\theta}_{1}^{o}
$$

where $\mathbf{B}_{1}, \mathbf{D}_{1}, \mathbf{h}_{1}$, and $\mathbf{G}_{1}$ are expressed as

$$
\begin{aligned}
\mathbf{B}_{1} & =\left[\begin{array}{ccc}
2 \mathbf{B} & \mathbf{O}_{(M-1) \times(M-1)} & \mathbf{O}_{(M-1) \times(M-1)} \\
\dot{\mathbf{B}} & \mathbf{B} & \mathbf{O}_{(M-1) \times(M-1)} \\
\ddot{\mathbf{B}} & 2 \dot{\mathbf{B}} & \mathbf{B}
\end{array}\right], \\
\mathbf{D}_{1} & =\left[\begin{array}{cc}
2 \mathbf{D} & \mathbf{O}_{(M-1) \times(3 M)} \\
\dot{\mathbf{D}} & \mathbf{D} \\
\mathbf{O}_{(M-1) \times(3 M)} & 2 \dot{\mathbf{D}}
\end{array}\right], \\
\mathbf{h}_{1} & =\left[\begin{array}{l}
\mathbf{h}_{1, r} \\
\mathbf{h}_{1, r} \\
\mathbf{h}_{1, \dot{r}}
\end{array}\right], \quad \mathbf{G}_{1}=\left[\begin{array}{l}
\mathbf{G}_{1, r} \\
\mathbf{G}_{1, r} \\
\mathbf{G}_{1, \dot{r}}
\end{array}\right]
\end{aligned}
$$

Now, (24) is a pseudo-linear equation in terms of three types of measurements with an unknown vector $\boldsymbol{\theta}_{1}^{o}$. Then, the WLS solution $\boldsymbol{\theta}_{1}^{o}$ is acquired from (24) as

$$
\hat{\boldsymbol{\theta}}_{1}=\left(\mathbf{G}_{1}^{T} \mathbf{W}_{1} \mathbf{G}_{1}\right)^{-1} \mathbf{G}_{1}^{T} \mathbf{W}_{1} \mathbf{h}_{1}
$$

where $\mathbf{W}_{1}$ denotes the weighting matrix. Based on the WLS theory and the matrix inversion Lemma [26], $\mathbf{W}_{1}$ can be clearly expressed as

$$
\begin{aligned}
\mathbf{W}_{1} & =E\left[\mathbf{\varepsilon}_{1} \mathbf{\varepsilon}_{1}^{T}\right]^{-1}=E\left[\left(\mathbf{B}_{1} \Delta \mathbf{\alpha}+\mathbf{D}_{1} \Delta \boldsymbol{\beta}\right)\left(\mathbf{B}_{1} \Delta \mathbf{\alpha}+\mathbf{D}_{1} \Delta \boldsymbol{\beta}\right)^{T}\right]^{-1} \\
& =\left(\mathbf{B}_{1} \mathbf{Q}_{\alpha} \mathbf{B}_{1}^{T}+\mathbf{D}_{1} \mathbf{Q}_{\beta} \mathbf{D}_{1}^{T}\right)^{-1} \\
& =\left(\mathbf{B}_{1} \mathbf{Q}_{\alpha} \mathbf{B}_{1}^{T}\right)^{-1}-\mathbf{B}_{1}^{-T} \mathbf{Q}_{\alpha}^{-1} \mathbf{B}_{1}^{-1} \mathbf{D}_{1}\left(\mathbf{Q}_{\beta}^{-1}+\mathbf{D}_{1}^{T} \mathbf{B}_{1}^{-T} \mathbf{Q}_{\alpha}^{-1} \mathbf{B}_{1}^{-1} \mathbf{D}_{1}\right) \mathbf{D}_{1}^{T} \mathbf{B}_{1}^{-T} \mathbf{Q}_{\alpha}^{-1} \mathbf{B}_{1}^{-1}
\end{aligned}
$$

Subtracting both sides of (26) by the true value $\boldsymbol{\theta}_{1}^{o}$ and using (24), we can obtain the estimation error of $\hat{\boldsymbol{\theta}}_{1}$

$$
\begin{aligned}
\Delta \boldsymbol{\theta}_{1} & =\hat{\boldsymbol{\theta}}_{1}-\boldsymbol{\theta}_{1}^{o}=\left(\mathbf{G}_{1}^{T} \mathbf{W}_{1} \mathbf{G}_{1}\right)^{-1} \mathbf{G}_{1}^{T} \mathbf{W}_{1} \mathbf{h}_{1}-\mathbf{\theta}_{1}^{o} \\
& =\left(\mathbf{G}_{1}^{T} \mathbf{W}_{1} \mathbf{G}_{1}\right)^{-1} \mathbf{G}_{1}^{T} \mathbf{W}_{1} \mathbf{h}_{1}-\left(\mathbf{G}_{1}^{T} \mathbf{W}_{1} \mathbf{G}_{1}\right)^{-1}\left(\mathbf{G}_{1}^{T} \mathbf{W}_{1} \mathbf{G}_{1}\right) \boldsymbol{\theta}_{1}^{o} \\
& =\left(\mathbf{G}_{1}^{T} \mathbf{W}_{1} \mathbf{G}_{1}\right)^{-1} \mathbf{G}_{1}^{T} \mathbf{W}_{1}\left(\mathbf{h}_{1}-\mathbf{G}_{1} \boldsymbol{\theta}_{1}^{o}\right)=\left(\mathbf{G}_{1}^{T} \mathbf{W}_{1} \mathbf{G}_{1}\right)^{-1} \mathbf{G}_{1}^{T} \mathbf{W}_{1} \boldsymbol{\varepsilon}_{1}
\end{aligned}
$$

Here, we need to use a basic assumption, usually used in prior studies into the measurement model [9-13, 17, 25], that the noise standard deviation relative to the true values of the TDOA, FDOA, and differential Doppler rate measurements is small. This assumption simplifies the algorithm development and analysis. It can be satisfied by increasing the observation period in obtaining the TDOA, FDOA, and differential Doppler rate. Under this assumption, the second-order noise terms of Eqs. 13,14 , and 15 tend to zero asymptotically as the measurement noise decreases so that $\boldsymbol{\varepsilon}_{1}$ is zero mean asymptotically at the true value of $\hat{\boldsymbol{\theta}}_{1}$. Hence, from the WLS theory [26], $\hat{\boldsymbol{\theta}}_{1}$ can be considered as approximately unbiased. In this case, the covariance $\hat{\boldsymbol{\theta}}_{1}$ can be approximated by 


$$
\begin{aligned}
\operatorname{cov}\left(\hat{\boldsymbol{\theta}}_{1}\right) & =E\left[\Delta \boldsymbol{\theta}_{1} \Delta \boldsymbol{\theta}_{1}^{T}\right]=E\left[\left(\mathbf{G}_{1}^{T} \mathbf{W}_{1} \mathbf{G}_{1}\right)^{-1} \mathbf{G}_{1}^{T} \mathbf{W}_{1} \varepsilon_{1} \mathbf{\varepsilon}_{1}^{T} \mathbf{W}_{1}^{T} \mathbf{G}_{1}\left(\mathbf{G}_{1}^{T} \mathbf{W}_{1} \mathbf{G}_{1}\right)^{-T}\right] \\
& =\left(\mathbf{G}_{1}^{T} \mathbf{W}_{1} \mathbf{G}_{1}\right)^{-1} \mathbf{G}_{1}^{T} \mathbf{W}_{1} E\left[\mathbf{\varepsilon}_{1} \mathbf{\varepsilon}_{1}^{T}\right] \mathbf{W}_{1}^{T} \mathbf{G}_{1}\left(\mathbf{G}_{1}^{T} \mathbf{W}_{1} \mathbf{G}_{1}\right)^{-T} \\
& =\left(\mathbf{G}_{1}^{T} \mathbf{W}_{1} \mathbf{G}_{1}\right)^{-1} \mathbf{G}_{1}^{T} \mathbf{W}_{1} \mathbf{W}_{1}^{-1} \mathbf{W}_{1}^{T} \mathbf{G}_{1}\left(\mathbf{G}_{1}^{T} \mathbf{W}_{1} \mathbf{G}_{1}\right)^{-T} \\
& =\left(\mathbf{G}_{1}^{T} \mathbf{W}_{1} \mathbf{G}_{1}\right)^{-1}\left(\mathbf{G}_{1}^{T} \mathbf{W}_{1} \mathbf{G}_{1}\right)\left(\mathbf{G}_{1}^{T} \mathbf{W}_{1} \mathbf{G}_{1}\right)^{-T}=\left(\mathbf{G}_{1}^{T} \mathbf{W}_{1} \mathbf{G}_{1}\right)
\end{aligned}
$$

where the weighting matrix $\mathbf{W}_{1}$ is a real symmetric matrix. It can be seen from (26) that the weighting matrix $\mathbf{W}_{1}$ is a function of source position and velocity, which is not available at the beginning in the first step of the proposed method. Therefore, let $\mathbf{W}_{1}=\mathbf{I}_{3(M-1) \times}$ $3(M-1)$ and use $\left(\mathbf{G}_{1}^{T} \mathbf{G}_{1}\right)^{-1} \mathbf{G}_{1}^{T} \mathbf{h}_{1}$ to obtain an initial estimation result for $\hat{\boldsymbol{\theta}}_{1}$. Then, use this initial result forms $\mathbf{W}_{1}$, and reuse $\mathbf{W}_{1}$ in (26) to produce a better estimate of $\hat{\boldsymbol{\theta}}_{1}$.

\subsection{Second WLS step}

Through the first step of the proposed method, the rough estimation result of source position and velocity can be found in $\hat{\boldsymbol{\theta}}_{1}(1: 3)$ and $\hat{\boldsymbol{\theta}}_{1}(5: 7)$. Note that, to rearrange the nonlinear measurement equations into linear forms in the first step, we have introduced extra additional variables $r_{1}^{o}, \dot{r}_{1}^{o}, \ddot{r}_{1}^{o}$, which are the functions of source position and velocity as seen in the following

$$
\begin{aligned}
& \boldsymbol{\theta}_{1}^{o 2}(4)=r_{1}^{o 2}=\left(\mathbf{u}^{o}-\mathbf{s}_{1}^{o}\right)^{T}\left(\mathbf{u}^{o}-\mathbf{s}_{1}^{o}\right) \\
& \boldsymbol{\theta}_{1}^{o}(4) \boldsymbol{\theta}_{1}^{o}(8)=r_{1}^{o} \dot{r}_{1}^{o}=\left(\dot{\mathbf{u}}^{o}-\dot{\mathbf{s}}_{1}^{o}\right)^{T}\left(\mathbf{u}^{o}-\mathbf{s}_{1}^{o}\right) \\
& \boldsymbol{\theta}_{1}^{o}(4) \boldsymbol{\theta}_{1}^{o}(9)+\boldsymbol{\theta}_{1}^{o 2}(8)=r_{1}^{o} \ddot{r}_{1}^{o}+\dot{r}_{1}^{o 2}=\left(\dot{\mathbf{u}}^{o}-\dot{\mathbf{s}}_{1}^{o}\right)^{T}\left(\dot{\mathbf{u}}^{o}-\dot{\mathbf{s}}_{1}^{o}\right)
\end{aligned}
$$

Hu et al. obtained the source location from Eq. (30) by utilizing the iteration process [17]. However, this method ignores the existence of the receiver location errors and has limited localization accuracy. Therefore, this section focuses on giving an algebraic solution without iteration to upgrade localization accuracy.

Let $\hat{\mathbf{u}}=\hat{\boldsymbol{\theta}}_{1}(1: 3), \hat{\dot{\mathbf{u}}}=\hat{\boldsymbol{\theta}}_{1}(5: 7), \hat{r}_{1}=\hat{\boldsymbol{\theta}}_{1}(4), \hat{\dot{r}}_{1}=\hat{\boldsymbol{\theta}}_{1}(8)$, and $\widehat{\vec{r}}_{1}=\hat{\boldsymbol{\theta}}_{1}(9)$, substitute $\mathbf{u}^{o}=\hat{\mathbf{u}}-\Delta \mathbf{u}, \dot{\mathbf{u}}^{o}=\widehat{\dot{\mathbf{u}}}-\Delta \dot{\mathbf{u}}, r_{1}^{o}$ $=\hat{r}_{1}-\Delta r_{1}, \dot{r}_{1}^{o}=\widehat{\dot{r}}_{1}-\Delta \dot{r}_{1}$, and $\ddot{r}_{1}^{o}=\widehat{\ddot{r}}_{1}-\Delta \ddot{r}_{1}$ into (30), ignore the second-order noise terms, and we have

$$
\begin{aligned}
& 2 \hat{\mathbf{u}} \Delta \mathbf{u}-2 \hat{r}_{1} \Delta r_{1}=\left(\hat{\mathbf{u}}^{T} \hat{\mathbf{u}}+\mathbf{s}_{1}^{o T} \mathbf{s}_{1}^{o}-\hat{r}_{1}^{2}\right)-2 \mathbf{s}_{1}^{\mathbf{s}_{1} T} \mathbf{u}^{o} \\
& \hat{\mathbf{u}} \Delta \mathbf{u}-\hat{\dot{r}}_{1} \Delta r_{1}+\hat{\mathbf{u}} \Delta \dot{\mathbf{u}}-\hat{r}_{1} \Delta \dot{r}_{1}=\left(\hat{\mathbf{u}} \mathbf{u} \hat{\mathbf{u}}+\widehat{\mathbf{s}}_{1}^{T} \mathbf{s}_{1}^{o}-\hat{\bar{r}}_{1} \hat{r}_{1}\right)-\dot{\mathbf{s}}_{1}^{o T} \mathbf{u}^{o}-\mathbf{s}_{1}^{o T} \dot{\mathbf{u}}^{o}
\end{aligned}
$$

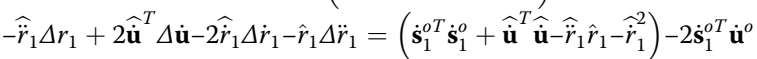

Then, replace $\mathbf{s}_{1}^{o}, \dot{\mathbf{s}}_{1}^{o}$ with $\mathbf{s}_{1}-\Delta \mathbf{s}_{1}$ and $\dot{\mathbf{s}}_{1}-\Delta \dot{\mathbf{s}}_{1}$, and three equations of (31) can be changed as

$$
\begin{aligned}
& 2 \hat{\mathbf{u}} \Delta \mathbf{u}-2 \hat{r}_{1} \Delta r_{1}-2\left(\hat{\mathbf{u}}-\mathbf{s}_{1}\right)^{T} \Delta \mathbf{s}_{1}=\left(\hat{\mathbf{u}}^{T} \hat{\mathbf{u}}+\mathbf{s}_{1}^{T} \mathbf{s}_{1}-\hat{r}_{1}^{2}\right)-2 \mathbf{s}_{1}^{T} \mathbf{u}^{o} \\
& \hat{\mathbf{u}} \Delta \mathbf{u}-\hat{r}_{1} \Delta r_{1}+\hat{\mathbf{u}} \Delta \dot{\mathbf{u}}-\hat{r}_{1} \Delta \dot{r}_{1}-\left(\widehat{\mathbf{u}}-\dot{\mathbf{s}}_{1}\right)^{T} \Delta \mathbf{s}_{1}-\left(\hat{\mathbf{u}}-\mathbf{s}_{1}\right)^{T} \Delta \dot{\mathbf{s}}_{1} \\
& =\left(\hat{\mathbf{u}}^{T} \hat{\mathbf{u}}+\widehat{\mathbf{s}}_{1}^{T} \mathbf{s}_{1}-\hat{\vec{r}}_{1} \hat{r}_{1}\right)-\dot{\mathbf{s}}_{1}^{T} \mathbf{u}^{o}-\mathbf{s}_{1}^{T} \dot{\mathbf{u}}^{o}-\hat{\vec{r}}_{1} \Delta r_{1} \\
& +2 \hat{\mathbf{u}}^{T} \Delta \dot{\mathbf{u}}-2 \hat{r}_{1} \Delta \dot{r}_{1}-\hat{r}_{1} \Delta \ddot{r}_{1}-2\left(\widehat{\hat{\mathbf{u}}}-\dot{\mathbf{s}}_{1}\right)^{T} \Delta \dot{\mathbf{s}}_{1}=\left(\dot{\mathbf{s}}_{1}^{T} \dot{\mathbf{s}}_{1}+\hat{\mathbf{u}}^{T} \hat{\mathbf{u}}-\hat{\vec{r}}_{1} \hat{r}_{1}-\hat{r}_{1}^{2}\right)-2 \dot{\mathbf{s}}_{1}^{T} \dot{\mathbf{u}}^{o}
\end{aligned}
$$

In addition, the final source position and velocity estimate should maintain as close as possible to the source location values obtained in the first WLS step by minimizing the errors of the following equations

$$
\begin{aligned}
& \Delta \mathbf{u}=\hat{\mathbf{u}}-\mathbf{u}^{o} \\
& \Delta \dot{\hat{\mathbf{u}}}=\dot{\mathbf{u}}^{o}
\end{aligned}
$$

Define $\boldsymbol{\theta}_{2}^{o}=\left[\mathbf{u}^{o}, \dot{\mathbf{u}}^{o T}\right]^{T}$, stack (32) and (33) together, and the matrix equation regarding $\boldsymbol{\theta}_{2}^{o}$ is

$$
\begin{aligned}
\boldsymbol{\varepsilon}_{2} & =\mathbf{B}_{2}\left[\Delta \mathbf{u}^{T}, \Delta r_{1}, \Delta \dot{\mathbf{u}}^{T}, \Delta \dot{r}_{1}, \Delta \dot{r}_{1}\right]^{T}+\mathbf{D}_{2} \Delta \boldsymbol{\beta} \\
& =\mathbf{h}_{2}-\mathbf{G}_{2} \boldsymbol{\theta}_{2}^{o}
\end{aligned}
$$

where

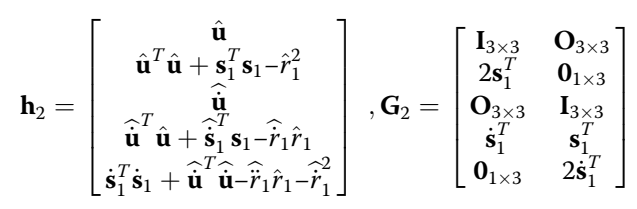

and

$$
\begin{aligned}
& \mathbf{B}_{2}=\left[\begin{array}{ccccc}
\mathbf{I}_{3 \times 3} & \mathbf{0}_{3 \times 1} & \mathbf{O}_{3 \times 3} & \mathbf{0}_{3 \times 1} & \mathbf{0}_{3 \times 1} \\
2 \hat{\mathbf{u}}^{T} & -2 \hat{r}_{1} & \mathbf{0}_{1 \times 3} & 0 & 0 \\
\mathbf{O}_{3 \times 3} & \mathbf{0}_{3 \times 1} & \mathbf{I}_{3 \times 3} & \mathbf{0}_{3 \times 1} & \mathbf{0}_{3 \times 1} \\
\hat{\mathbf{u}}^{T} & -\hat{\hat{r}}_{1} & \hat{\mathbf{u}}^{T} & -\hat{r}_{1} & 0 \\
\mathbf{0}_{1 \times 3} & -\hat{\tilde{r}}_{1} & 2 \hat{\mathbf{u}}^{T} & -2 \hat{r}_{1} & -\hat{r}_{1}
\end{array}\right], \\
& \mathbf{D}_{2}=-\left[\begin{array}{cccc}
\mathbf{O}_{3 \times 6 M} & & & \\
2\left(\hat{\mathbf{u}}-\mathbf{s}_{1}\right)^{T} & \mathbf{0}_{1 \times(6 M-3)} & & \\
\mathbf{O}_{3 \times 6 M} & & & \\
\left(\hat{\hat{\mathbf{u}}}-\dot{\mathbf{s}}_{1}\right)^{T} & \mathbf{0}_{1 \times(3 M-3)} & \left(\hat{\mathbf{u}}-\mathbf{s}_{1}\right)^{T} & \mathbf{0}_{1 \times(3 M-3)} \\
\mathbf{0}_{1 \times 3} & \mathbf{0}_{1 \times(3 M-3)} & 2\left(\widehat{\mathbf{u}}-\dot{\mathbf{s}}_{1}\right)^{T} & \mathbf{0}_{1 \times(3 M-3)}
\end{array}\right]
\end{aligned}
$$

The WLS solution $\boldsymbol{\theta}_{2}^{o}$ is acquired from (34) as

$$
\hat{\boldsymbol{\theta}}_{2}=\left(\mathbf{G}_{2}^{T} \mathbf{W}_{2} \mathbf{G}_{2}\right)^{-1} \mathbf{G}_{2}^{T} \mathbf{W}_{2} \mathbf{h}_{2}
$$

The weighting matrix $\mathbf{W}_{2}$ is

$$
\begin{aligned}
\mathbf{W}_{2} & =E\left[\boldsymbol{\varepsilon}_{2} \boldsymbol{\varepsilon}_{2}^{T}\right]^{-1} \\
& =E\left[\left(\mathbf{B}_{2} \Delta \boldsymbol{\theta}_{1}+\mathbf{D}_{2} \Delta \boldsymbol{\beta}\right)\left(\mathbf{B}_{2} \Delta \boldsymbol{\theta}_{1}+\mathbf{D}_{2} \Delta \boldsymbol{\beta}\right)^{T}\right]^{-1}
\end{aligned}
$$

Due to 


$$
\begin{aligned}
E\left[\Delta \boldsymbol{\theta}_{1} \Delta \boldsymbol{\beta}^{T}\right] & =E\left[\left(\mathbf{G}_{1}^{T} \mathbf{W}_{1} \mathbf{G}_{1}\right)^{-1} \mathbf{G}_{1}^{T} \mathbf{W}_{1} \mathbf{\varepsilon}_{1} \Delta \boldsymbol{\beta}^{T}\right] \\
& =E\left[\left(\mathbf{G}_{1}^{T} \mathbf{W}_{1} \mathbf{G}_{1}\right)^{-1} \mathbf{G}_{1}^{T} \mathbf{W}_{1}\left(\mathbf{B}_{1} \Delta \boldsymbol{\alpha}+\mathbf{D}_{1} \Delta \boldsymbol{\beta}\right) \Delta \boldsymbol{\beta}^{T}\right] \\
& =\left(\mathbf{G}_{1}^{T} \mathbf{W}_{1} \mathbf{G}_{1}\right)^{-1} \mathbf{G}_{1}^{T} \mathbf{W}_{1} \mathbf{D}_{1} E\left[\Delta \boldsymbol{\beta} \Delta \boldsymbol{\beta}^{T}\right] \\
& =\left(\mathbf{G}_{1}^{T} \mathbf{W}_{1} \mathbf{G}_{1}\right)^{-1} \mathbf{G}_{1}^{T} \mathbf{W}_{1} \mathbf{D}_{1} \mathbf{Q}_{\beta}
\end{aligned}
$$

$\mathbf{W}_{2}$ can be specifically expressed as

$$
\begin{aligned}
\mathbf{W}_{2}=\left(\mathbf{B}_{2} \operatorname{cov}\left(\boldsymbol{\theta}_{1}\right) \mathbf{B}_{2}^{T}+\mathbf{D}_{2} \mathbf{Q}_{\beta} \mathbf{D}_{2}^{T}\right. & +\mathbf{B}_{2}\left(\mathbf{G}_{1}^{T} \mathbf{W}_{1} \mathbf{G}_{1}\right)^{-1} \mathbf{G}_{1}^{T} \mathbf{W}_{1} \mathbf{D}_{1} \mathbf{Q}_{\beta} \mathbf{D}_{2}^{T} \\
& \left.+\mathbf{D}_{2} \mathbf{Q}_{\beta} \mathbf{D}_{1}^{T} \mathbf{W}_{1} \mathbf{G}_{1}\left(\mathbf{G}_{1}^{T} \mathbf{W}_{1} \mathbf{G}_{1}\right)^{-1} \mathbf{B}_{2}^{T}\right)^{-1}
\end{aligned}
$$

In summary, the steps for estimating the source position $\mathbf{u}^{o}$ and velocity $\dot{\mathbf{u}}^{o}$ in this paper are listed as follows:

(i) Set $\mathbf{W}_{1}=\mathbf{I}_{3(M-1) \times 3(M-1)}$ and use $\left(\mathbf{G}_{1}^{T} \mathbf{G}_{1}\right)^{-1} \mathbf{G}_{1}^{T} \mathbf{h}_{1}$ to obtain an initial estimate of $\hat{\boldsymbol{\theta}}_{1}$;

(ii) Utilize this initial estimate to form the approximate $\mathbf{W}_{1}$ and reuse (26) to update $\hat{\boldsymbol{\theta}}_{1}$;

(iii) Find $\hat{\boldsymbol{\theta}}_{2}$ from (37), where $\mathbf{W}_{2}$ is given in (40).

(iv) The final source position and velocity estimate can be obtained from $\hat{\boldsymbol{\theta}}_{2}(1: 3)$ and $\hat{\boldsymbol{\theta}}_{2}(4: 6)$.

\section{CRLB and performance analysis}

\subsection{CRLB}

CRLB is the lowest possible variance achieved by an unbiased linear estimator and often regarded as a benchmark for performance evaluation. This section gives a new CRLB derivation of source position and velocity based on TDOA, FDOA, and differential Doppler rate with receiver location errors, which is different from [10, 17, 25]. As postulated in Section 2, three types of positioning measurement noise $\Delta \boldsymbol{\alpha}$ and receiver location error noise $\Delta \boldsymbol{\beta}$ obey the Gaussian distribution with zero means which are independent of each other. Thus, according to the assumption mentioned above, the logarithm of the joint density function of $\boldsymbol{\alpha}$ and $\boldsymbol{\beta}$ parameterized on $\varphi^{\circ}$ is

$$
\begin{aligned}
\ln f\left(\boldsymbol{\alpha}, \boldsymbol{\beta}, \boldsymbol{\phi}^{o}\right) & =\ln f\left(\boldsymbol{\alpha}, \boldsymbol{\phi}^{o}\right)+\ln f\left(\boldsymbol{\beta}, \boldsymbol{\phi}^{o}\right) \\
& =K-\frac{1}{2}\left[\boldsymbol{\alpha}-\boldsymbol{\alpha}^{o}\right]^{T} \mathbf{Q}_{\boldsymbol{\alpha}}{ }^{-1}\left[\boldsymbol{\alpha}-\boldsymbol{\alpha}^{o}\right]-\frac{1}{2}\left[\boldsymbol{\beta}-\boldsymbol{\beta}^{o}\right]^{T} \mathbf{Q}_{\beta}{ }^{-1}\left[\boldsymbol{\beta}-\boldsymbol{\beta}^{o}\right]
\end{aligned}
$$

where $\left.K=-1 / 2 \ln \left((2 \pi)^{3(M-1)} \mid \mathbf{Q}_{\alpha}\right\rfloor\right)-1 / 2 \ln \left((2 \pi)^{6 M}\left|\mathbf{Q}_{\boldsymbol{\beta}}\right|\right)$ is a constant, and $\boldsymbol{\phi}^{o}=\left[\boldsymbol{\theta}_{2}^{o T}, \boldsymbol{\beta}^{o T}\right]^{T}$. Based on (41), the CRLB for $\phi^{\circ}$ is given by

$$
\operatorname{CRLB}\left(\boldsymbol{\phi}^{o}\right)=-E\left[\left(\frac{\partial^{2} \ln f\left(\boldsymbol{\alpha}, \boldsymbol{\beta}, \boldsymbol{\phi}^{o}\right)}{\partial \boldsymbol{\phi}^{o} \partial \boldsymbol{\phi}^{o T}}\right)\right]_{(6 M+6) \times(6 M+6)}^{-1}
$$

where $\operatorname{CRLB}\left(\phi^{o}\right)$ denotes the Cramer-Rao lower bound of $\phi^{o}$. It can be seen from (42) that $\operatorname{CRLB}\left(\phi^{\circ}\right)$ is a $6 M+$ 6 square matrix, in which only the upper left $6 \times 6$ submatrix corresponding to the CRLB is of interest to us. For the convenience of expression, we convert (42) into submatrix form shown as

$$
\operatorname{CRLB}\left(\boldsymbol{\phi}^{o}\right)=\left[\begin{array}{ll}
\boldsymbol{R}_{1} & \boldsymbol{R}_{2} \\
\boldsymbol{R}_{2}^{T} & \boldsymbol{R}_{3}
\end{array}\right]^{-1}
$$

where

$$
\begin{aligned}
& \boldsymbol{R}_{1}=E\left[\left(\frac{\partial^{2} \ln f\left(\boldsymbol{\alpha}, \boldsymbol{\beta}, \boldsymbol{\phi}^{o}\right)}{\partial \boldsymbol{\theta}_{2}^{o} \partial \boldsymbol{\theta}_{2}^{o T}}\right)\right]=\left(\frac{\partial \mathbf{\alpha}^{o}}{\partial \boldsymbol{\theta}_{2}^{o T}}\right)^{T} \mathbf{Q}_{\boldsymbol{\alpha}}^{-1} \frac{\partial \mathbf{\alpha}^{o}}{\partial \boldsymbol{\theta}_{2}^{o T}} \\
& \boldsymbol{R}_{2}=E\left[\left(\frac{\partial^{2} \ln f\left(\boldsymbol{\alpha}, \boldsymbol{\beta}, \boldsymbol{\phi}^{o}\right)}{\partial \boldsymbol{\theta}_{2}^{o} \partial \boldsymbol{\beta}^{o T}}\right)\right]=\left(\frac{\partial \mathbf{\alpha}^{o}}{\partial \boldsymbol{\theta}_{2}^{o T}}\right)^{T} \boldsymbol{Q}_{\boldsymbol{\alpha}}^{-1} \frac{\partial \mathbf{\alpha}^{o}}{\partial \boldsymbol{\beta}^{o T}} \\
& \boldsymbol{R}_{3}=E\left[\left(\frac{\partial^{2} \ln f\left(\boldsymbol{\alpha}, \boldsymbol{\beta}, \boldsymbol{\phi}^{o}\right)}{\partial \boldsymbol{\beta}^{o} \partial \boldsymbol{\beta}^{o T}}\right)\right]=\left(\frac{\partial \mathbf{\alpha}^{o}}{\partial \boldsymbol{\beta}^{o T}}\right)^{T} \mathbf{Q}_{\boldsymbol{\alpha}}^{-1} \frac{\partial \mathbf{\alpha}^{o}}{\partial \boldsymbol{\beta}^{o T}}+Q_{\boldsymbol{\beta}}^{-1}
\end{aligned}
$$

The partial derivatives $\partial \mathbf{\alpha}^{o} / \partial \boldsymbol{\theta}_{2}^{o T}$ and $\partial \mathbf{\alpha}^{o} / \partial \boldsymbol{\beta}^{o T}$ are given in Appendix 1 and 2, respectively. According to the partitioned matrix inversion formula in [26], (44) can be rewritten as

$$
\begin{aligned}
\boldsymbol{C R L B}\left(\boldsymbol{\theta}_{2}^{o}\right) & =\left(\boldsymbol{R}_{1}-\boldsymbol{R}_{2} \boldsymbol{R}_{3}^{-1} \boldsymbol{R}_{2}^{T}\right)^{-1} \\
& =\boldsymbol{R}_{1}^{-1}+\boldsymbol{R}_{1}^{-1} \boldsymbol{R}_{2}\left(\boldsymbol{R}_{3}-\boldsymbol{R}_{2}^{T} \boldsymbol{R}_{1}^{-1} \boldsymbol{R}_{2}\right)^{-1} \boldsymbol{R}_{2}^{T} \boldsymbol{R}_{1}^{-1}
\end{aligned}
$$

It is important to emphasize that, in (45), $\boldsymbol{R}_{1}^{-1}$ is the CRLB of the source position and velocity without receiver location errors derived in [17]. Since $\left(\boldsymbol{R}_{3}-\boldsymbol{R}_{2}^{T} \boldsymbol{R}_{1}^{-1}\right.$ $\boldsymbol{R}_{2}$ ) is the positive definite matrix, the second term in (45) stands for the increase in CRLB in the presence of receiver location errors. In the subsequent numerical simulation section, the CRLB with receiver location errors and that without receiver location errors will be compared numerically to verify this analysis.

\subsection{Performance analysis}

This section gives a rigorously theoretical derivation regarding the relationship between the covariance matrix of the proposed method and the CRLB derived in Section 5.1. According to the definition of $\hat{\boldsymbol{\theta}}_{2}$ below (33), the covariance of the proposed method equals to the covariance of $\hat{\boldsymbol{\theta}}_{2}$. Hence, to analyze the covariance of the second-step solution $\hat{\boldsymbol{\theta}}_{2}$, we subtract both sides of (37) by $\boldsymbol{\theta}_{2}^{o}=\left(\mathbf{G}_{2}^{T} \mathbf{W}_{2} \mathbf{G}_{2}\right)^{-1} \mathbf{G}_{2}^{T} \mathbf{W}_{2} \mathbf{G}_{2} \boldsymbol{\theta}_{2}^{o}$ and use (34) yielding

$$
\Delta \boldsymbol{\theta}_{2}=\hat{\boldsymbol{\theta}}_{2}-\boldsymbol{\theta}_{2}^{o}=\left(\mathbf{G}_{2}^{T} \mathbf{W}_{2} \mathbf{G}_{2}\right)^{-1} \mathbf{G}_{2}^{T} \mathbf{W}_{2} \boldsymbol{\varepsilon}_{2}
$$

It is easy to deduce that, under small noise condition, the second-step solution $\hat{\boldsymbol{\theta}}_{2}$ is approximately unbiased and its covariance matrix can be approximately expressed by 


$$
\operatorname{cov}\left(\hat{\boldsymbol{\theta}}_{2}\right) \simeq\left(\mathbf{G}_{2}^{T} \mathbf{W}_{2} \mathbf{G}_{2}\right)^{-1}
$$

Then, replace $\mathbf{W}_{1}$ and $\operatorname{cov}\left(\hat{\boldsymbol{\theta}}_{1}\right)$ in (40) by (27) and (29), substitute $\mathbf{W}_{2}$ in (47) by (40), the inverse of (47) can be further approximately rewritten as

$$
\operatorname{cov}\left(\hat{\boldsymbol{\theta}}_{2}\right)^{-1} \simeq \mathbf{G}_{3}^{T} \mathbf{Q}_{\alpha}^{-1} \mathbf{G}_{3}-\mathbf{G}_{3}^{T} \mathbf{Q}_{\alpha}^{-1} \mathbf{G}_{4}\left(\mathbf{Q}_{\boldsymbol{\beta}}^{-1}+\mathbf{G}_{4}^{T} \mathbf{Q}_{\alpha}^{-1} \mathbf{G}_{4}\right) \mathbf{G}_{4}^{T} \mathbf{Q}_{\alpha}^{-1} \mathbf{G}_{3}
$$

where

$$
\begin{aligned}
& \mathbf{G}_{3}=\mathbf{B}_{1}^{-1} \mathbf{G}_{1} \mathbf{B}_{2}^{-1} \mathbf{G}_{2} \\
& \mathbf{G}_{4}=\mathbf{B}_{1}^{-1} \mathbf{D}_{1}
\end{aligned}
$$

Under the following assumptions about small measurement noise and receiver location errors $[10,25]$

$$
\begin{array}{ll}
\Delta r_{i 1} / r_{i 1}^{o} \simeq 0, & \left\|\Delta \mathbf{s}_{i}\right\| / r_{i 1}^{o} \simeq 0 \\
\Delta \dot{r}_{i 1} \dot{r}_{i 1}^{o} \simeq 0, & \left\|\Delta \dot{s}_{i}\right\| / \dot{r}_{i 1}^{o} \simeq 0 \\
\Delta \ddot{r}_{i 1} / \ddot{r}_{i 1}^{o} \simeq 0, & \left\|\Delta \ddot{\mathbf{s}}_{i}\right\| / \ddot{r}_{i 1}^{o} \simeq 0
\end{array}
$$

for $i=2,3, \ldots, M$, we obtain

$$
\mathbf{G}_{3}=\frac{\partial \boldsymbol{\alpha}^{o}}{\partial \boldsymbol{\theta}_{2}^{o T}}, \quad \mathbf{G}_{4}=\frac{\partial \boldsymbol{\alpha}^{o}}{\partial \boldsymbol{\beta}^{o T}}
$$

Observe that the covariance matrix of the proposed method in (48) and the CRLB in (45) are of the same form. Under assumptions that the measurement noise and receiver location errors are sufficiently small, we can conclude that the CRLB is achieved.

\section{Simulation results}

In this section, numerical simulations are performed to evaluate the theoretical development of the proposed method. Consider a localization scenario including five moving receivers used to determine one moving source and their position and velocity are listed in Table 1 . We assume that the source moves in a circle with radius $R$, height $400 \mathrm{~m}$ and absolute speed $30 \mathrm{~m} / \mathrm{s}$. The orientation of the velocity is vertical to the line from the circle center to source position. Therefore, the position and velocity of the source are respectively expressed as

$$
\begin{aligned}
\mathbf{u}^{o} & =[R \cos \phi, R \sin \phi, 400]^{T} \mathrm{~m} \\
\dot{\mathbf{u}}^{o} & =[-30 \sin \phi, 30 \cos \phi, 0]^{T} \mathrm{~m} / \mathrm{s}, \quad \phi \in[0,2 \pi]
\end{aligned}
$$

Table 1 Position and velocity of the receivers

\begin{tabular}{lllllll}
\hline Receiver no. $i$ & $x_{i}(\mathrm{~m})$ & $y_{i}(\mathrm{~m})$ & $z_{i}(\mathrm{~m})$ & $\dot{x}_{i}(\mathrm{~m} / \mathrm{s})$ & $\dot{y}_{i}(\mathrm{~m} / \mathrm{s})$ & $\dot{z}_{i}(\mathrm{~m} / \mathrm{s})$ \\
\hline 1 & 0 & 0 & -100 & 0 & 0 & 0 \\
2 & 0 & 300 & 0 & -20 & 0 & 0 \\
3 & -300 & 0 & 0 & 0 & -20 & 0 \\
4 & 0 & -300 & 0 & 20 & 0 & 0 \\
5 & 300 & 0 & 0 & 0 & 20 & 0 \\
\hline
\end{tabular}

where $R$ and $\phi$ are the moving radius and the azimuth angle.

The simulation results illustrate the performance of the proposed solution for different measurement noise levels, which can be obtained from the following equations [19].

$$
\begin{aligned}
\sigma_{\tau} & =\frac{1}{B_{s}} \frac{1}{\sqrt{B_{n} T}} \frac{1}{\sqrt{\mathrm{SNR}}} \\
\sigma_{f} & =\frac{\sqrt{3}}{\pi T} \frac{1}{\sqrt{B_{n} T}} \frac{1}{\sqrt{\mathrm{SNR}}} \\
\sigma_{f} & =\frac{2 \sqrt{45}}{\pi T^{2}} \frac{1}{\sqrt{B_{n} T}} \frac{1}{\sqrt{\mathrm{SNR}}}
\end{aligned}
$$

where $\sigma_{t}, \sigma_{f}$ and $\sigma_{\dot{f}}$ respectively denote the standard deviation of TDOA, FDOA, and differential Doppler rate measurement error. $B_{s}$ and $B_{n}$ are respectively the signal and the noise bandwidth, $T$ is the integration time, and SNR is the input signal-to-noise ratio. The covariance matrix of measurement noises is

$$
Q_{\alpha}=\left[\begin{array}{lll}
\boldsymbol{Q}_{\mathbf{r}} & & \\
& \boldsymbol{Q}_{\dot{\mathbf{r}}} & \\
& & \boldsymbol{Q}_{\dot{\mathbf{r}}}
\end{array}\right]_{3(M-1) \times 3(M-1)}
$$

where

$$
\begin{aligned}
& \boldsymbol{Q}_{\mathbf{r}}=\left(c \sigma_{\tau}\right)^{2} \mathbf{V}_{(M-1) \times(M-1)} \\
& \boldsymbol{Q}_{\dot{\mathbf{r}}}=\left(\lambda \sigma_{f}\right)^{2} \mathbf{V}_{(M-1) \times(M-1)} \\
& \boldsymbol{Q}_{\ddot{\mathbf{r}}}=\left(\lambda \sigma_{\ddot{f}}\right)^{2} \mathbf{V}_{(M-1) \times(M-1)}
\end{aligned}
$$

The noisy receiver locations are simulated by adding zero mean Gaussian noises to the true value with covariance matrix

$$
\mathbf{Q}_{\boldsymbol{\beta}}=\left[\begin{array}{ll}
\mathbf{Q}_{s} & \\
& \mathbf{Q}_{\dot{s}}
\end{array}\right]_{6 M \times 6 M}
$$

where $\mathbf{Q}_{s}=\sigma_{s}^{2} \mathbf{V}_{3 M \times 3 M}$ and $\mathbf{Q}_{\dot{s}}=0.1 \mathbf{Q}_{s}$ with $\sigma_{s}$ representing the receiver position error level. In the following simulations, the received signal is assumed to be binary phase shift keying modulated, and we fix $f_{c}=3 \mathrm{GHz}, B_{s}=$ $100 \mathrm{KHz}, T=0.1 \mathrm{~s}$, and $B_{n}=3 B_{s}$, and vary SNR and $\sigma_{s}$ by small steps to characterize the performance of the proposed solution for different measurement noise levels and receiver position error level. The $\mathrm{dBm}$ means the $\mathrm{dB}$ meters in the following. The estimation accuracy is evaluated in terms of the RMSE, which is defined as

$$
\begin{aligned}
\operatorname{RMSE}\left(\mathbf{u}^{o}\right) & =\sqrt{\sum_{l=1}^{L}\left\|\mathbf{u}^{o}-\mathbf{u}_{l}\right\|^{2} / L} \\
\operatorname{RMSE}\left(\dot{\mathbf{u}}^{o}\right) & =\sqrt{\sum_{l=1}^{L}\left\|\dot{\mathbf{u}}^{o}-\dot{\mathbf{u}}_{l}\right\|^{2} / L}
\end{aligned}
$$

where $\mathbf{u}^{o}$ and $\dot{\mathbf{u}}^{o}$ stand for the true value of the source position and velocity, and each Monte Carlo estimate of 
source position and velocity is expressed as $\mathbf{u}_{l}$ and $\dot{\mathbf{u}}_{l}$. Each simulation includes $L=5000$ independent Monte Carlo runs.

\subsection{CRLB comparison}

In this section, we compare the CRLB derived in Section 4.1 with the CRLB in [17] to illustrate how sensitive the localization accuracy is with regard to the receiver location errors and SNR. We set $R=500 \mathrm{~m}$ and $\phi=\pi / 3$ in (52), thereby $\mathbf{u}^{o}=[250,250 \sqrt{3}, 400] \mathrm{m}$ and $\dot{\mathbf{u}}^{o}=[15 \sqrt{3}, 15,0]^{T} \mathrm{~m} / \mathrm{s}$.

Firstly, Fig. 1 depicts the CRLBs as $\sigma_{s}$ changes from 20 to $30 \mathrm{dBm}$ and $\mathrm{SNR}=10 \mathrm{~dB}$. Based on (53), the calculated accuracy of TDOA, FDOA, and differential Doppler rate is $\sigma_{\tau}=19 \mathrm{~ns}, \sigma_{f}=0.01 \mathrm{~Hz}$, and $\sigma_{\dot{f}}=0.8 \mathrm{~Hz} / \mathrm{s}$, respectively. The two covariance matrixes of measurement noises and receiver locations noise are obtained by (54) and (56). It can be seen that the gap between the two types of CRLBs becomes more and more obvious with the increase of $\sigma_{s}$. For the CRLB with receiver location error derived in Section 4.1, there are relatively increase compared with the CRLB without receiver location errors in [17] at $\sigma_{s}=3.16 \mathrm{~m} \quad\left(20 \log _{10}\left(\sigma_{s}\right)=\right.$ $10 \mathrm{~dB})$, which is not rare in practice $[27,28]$. This implies that the existence of the receiver location error can deteriorate the estimation accuracy of source position and velocity.

Secondly, we fix the receiver location error at $\sigma_{s}=$ $10^{0.5} \mathrm{~m}$ and compare two types of CRLBs as SNR changes from $-20 \mathrm{~dB}$ to $30 \mathrm{~dB}$ shown in Fig. 2. It is readily seen that the CRLB with receiver location error is above the one without receiver location error. When SNR is low, the localization accuracy is mainly dominated by SNR and is not appreciably affected by the receiver location errors. Therefore, these two types of CRLBs are approximately equal. However, as SNR increases, the negative influence of the receiver location error on estimation accuracy gradually appears and this error limits further improvement of performance at high SNR, which signifies again the necessity of taking into consideration the receiver location errors during the design of localization methods for moving source localization, at least in the CRLB sense.

\subsection{Robustness comparison}

This section compares the robustness of the proposed method with that of TSWLS method [25], Hu's method [17], as well as the three types of CRLBs [17, 25] via Monte Carlo simulations, as $\phi$ changes from 0 to $2 \pi$ in (52). Moreover, to investigate the robustness of the proposed method completely, we set four types of sources with different radius shown in Fig. 3. SNR $=10 \mathrm{~dB}$ and $\sigma_{s}=10^{0.5} \mathrm{~m}$; the two covariance matrixes of measurement noises and receiver locations noise are also calculated by (54) and (56). Other simulation conditions are the same as that in the previous one.

Figure 4 shows the robustness comparison among different methods with four different radiuses $(R=20 \mathrm{~m}$, $500 \mathrm{~m}, 1000 \mathrm{~m}, 4000 \mathrm{~m})$. The TSWLS method [25], Hu's method [17], and their corresponding CRLBs [17, 25] are plotted to compare with the proposed method. The experiment results and analysis are listed as follows:

1. The RMSE curves of source position and velocity with $R=500 \mathrm{~m}$ and $R=1000 \mathrm{~m}$ are shown in Fig. 4c, $\mathrm{d}$, e, and $\mathrm{f}$. This is the scenario where the source is in the moderate distance from the receivers. It can be seen that due to the existence of receiver location errors, Hu's method cannot attain its CRLB and perform lower localization accuracy compared with the proposed method. Moreover, as $\phi$ changes, the proposed method always performs robustly while the RMSE of TSWLS method increases sharply at some values of $\phi$ even at high SNR due to its rank deficiency problem in its second step. More specifically, the matrix $\mathbf{B}_{2}$ of the TSWLS method in [25], defined as

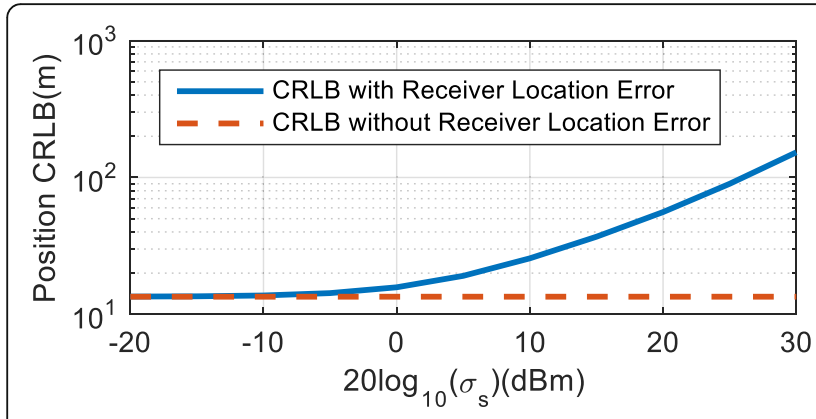

(a) Position CRLB

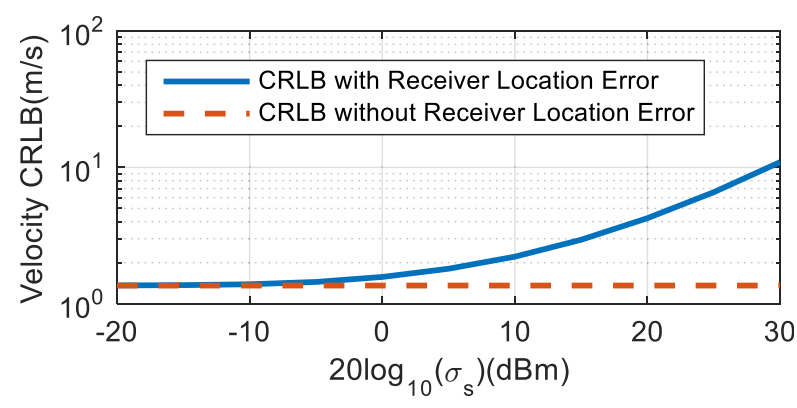

(b) Velocity CRLB

Fig. 1 CRLB comparison versus receiver location error when $S N R=10 \mathrm{~dB}$. a Position CRLB. b Velocity CRLB 


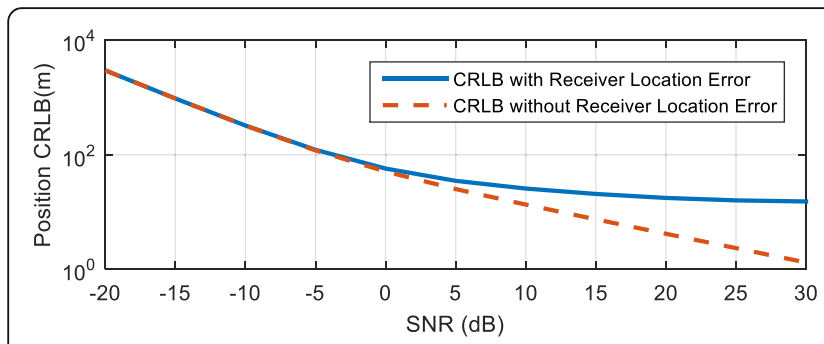

(a) Position CRLB

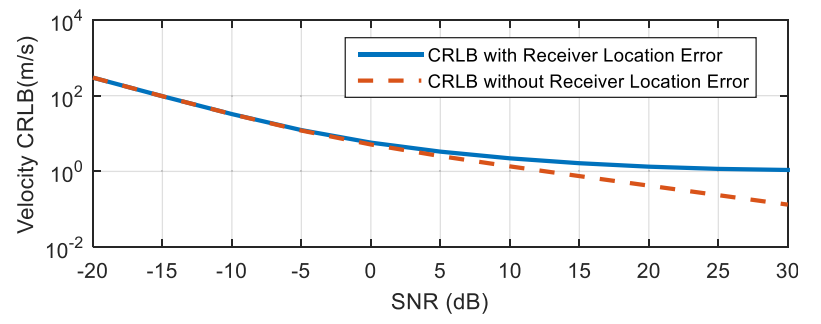

(b) Velocity CRLB

Fig. 2 CRLB comparison versus SNR when $\sigma_{s}=10^{0.5} \mathrm{~m}$. a Position CRLB. b Velocity CRLB

$$
\left[\begin{array}{cccc}
2 \operatorname{diag}\left\{\mathbf{u}^{o}-\mathbf{s}_{1}^{o}\right\} & \mathbf{0}_{3 \times 1} & \mathbf{O}_{3 \times 3} & \mathbf{0}_{3 \times 1} \\
\mathbf{0}_{1 \times 3} & 2 r_{1}^{o} & \mathbf{0}_{1 \times 3} & 0 \\
\operatorname{diag}\left\{\dot{\mathbf{u}}^{o}-\dot{\mathbf{s}}_{1}^{o}\right\} & \mathbf{0}_{3 \times 1} & \operatorname{diag}\left\{\mathbf{u}^{o}-\mathbf{s}_{1}^{o}\right\} & \mathbf{0}_{3 \times 1} \\
\mathbf{0}_{1 \times 3} & \dot{r}_{1}^{o} & \mathbf{0}_{1 \times 3} & 2 r_{1}^{o}
\end{array}\right]
$$

is not full-rank when $\phi=k \pi / 2, k=0,1, \ldots, 4$ in current localization scenario. For instance, when $\phi=\pi / 2$ and $R$ $=500 \mathrm{~m},\left[\mathbf{u}^{o}-\mathbf{s}_{1}^{o}\right]^{T}$ equals to $[0,500,500]^{T}$ and the matrix (58) is not full-rank, which may lead to obtaining an inaccurate estimation result when solving the inverse of the matrix (58). On the contrary, the proposed method can efficiently avoid this serious problem and always attains the CRLB well.

2. The RMSE curves of source position and velocity with $R=20 \mathrm{~m}$ are shown in Fig. $4 \mathrm{a}$ and b. This is the scenario where the source approaches receivers. It can be observed that the TSWLS method and Hu's method in [25] cannot give an accurate source location, while the proposed method still works in this near-field scenario and the accuracy curve achieves CRLB very well. The reason why the TSWLS method fails still lies in (58). For example, when $R=20 \mathrm{~m},\left[\mathbf{u}^{o}-\mathbf{s}_{1}^{o}\right]^{T}$ is $[20 \cos \phi, 20 \sin \phi, 500]^{T}$. The ratio between the maximum element and the minimum element is at least 25 . Therefore, the rank deficiency problem of the TSWLS method still easily occurs in this condition.

3. The RMSE curves of source position and velocity with $R=4000 \mathrm{~m}$ are shown in Fig. $4 \mathrm{~g}$ and $\mathrm{h}$. This is the scenario where the source is far from the receivers. With the change of $\phi$, the RMSE of the proposed method still always matches its CRLB without any fail point when the source moves in the far-field scenario, while Hu's method cannot attain its CRLB under the condition of existing receiver location errors. Moreover, the TSWLS method does not work and is not suitable for locating the far-field source, even after considering the receiver location errors in the method.

In summary, when the source is close to or far from the receivers, the estimation accuracy of the TSWLS method is poor. In addition, even if the source moves to a moderate distance from the receivers, this method

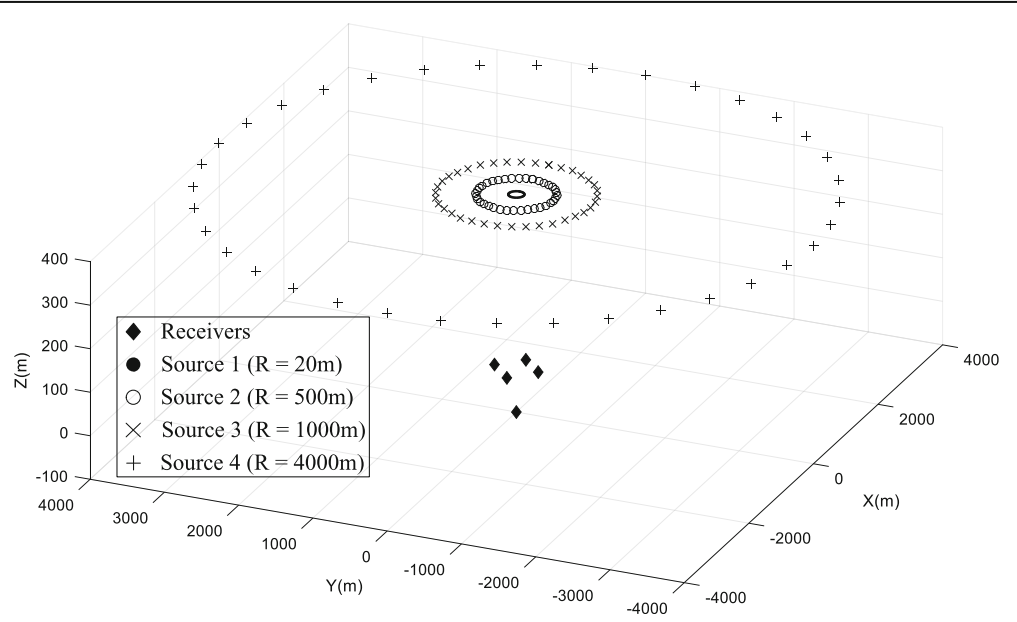

Fig. 3 Localization geometry of receivers and sources (circular motion) 


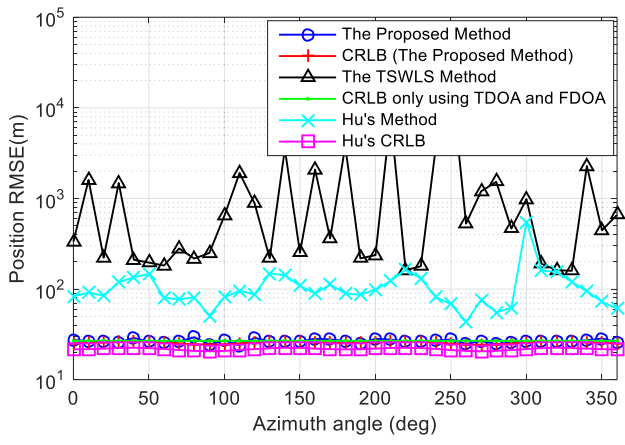

(a) RMSEs of position with radius of $20 \mathrm{~m}$

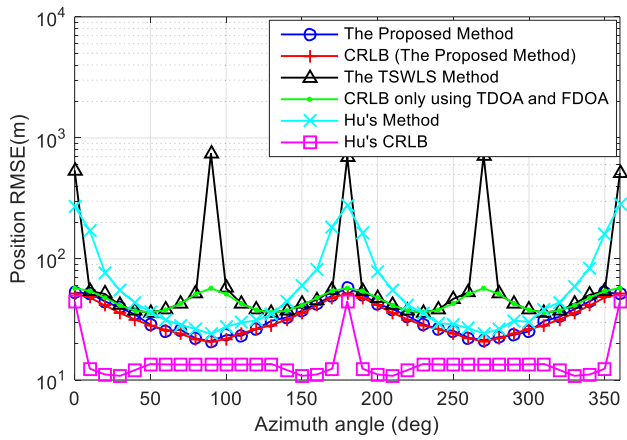

(c) RMSEs of position with radius of $500 \mathrm{~m}$

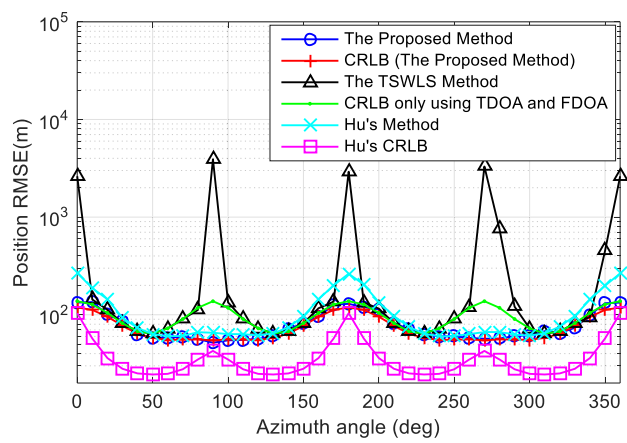

(e) RMSEs of position with radius of $1000 \mathrm{~m}$

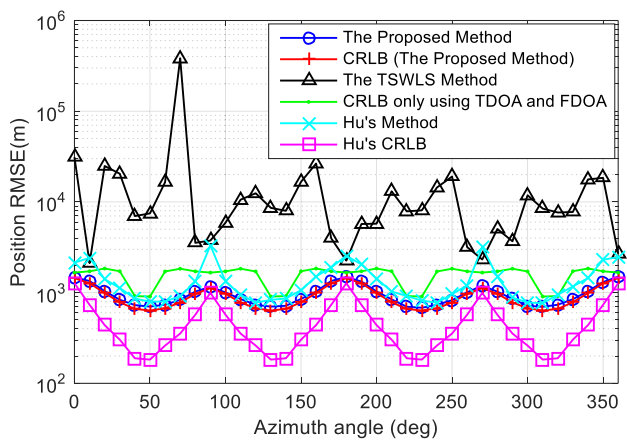

(g) RMSEs of position with radius of $4000 \mathrm{~m}$

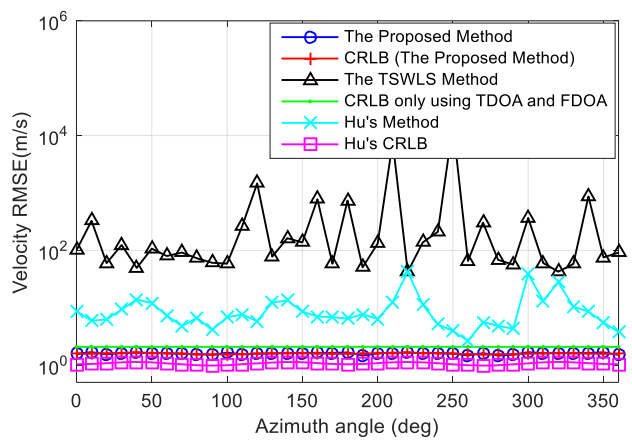

(b) RMSEs of velocity with radius of $20 \mathrm{~m}$

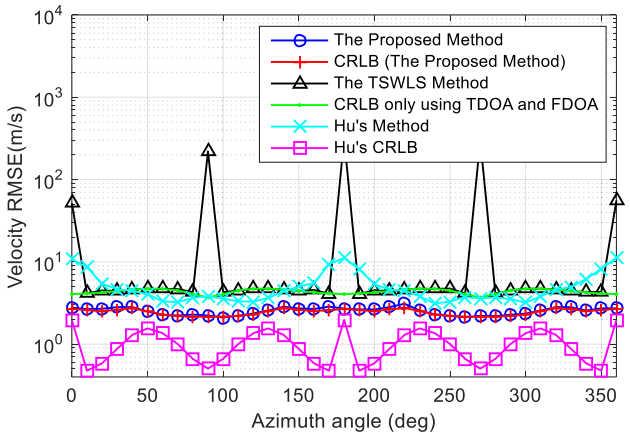

(d) RMSEs of velocity with radius of 500m

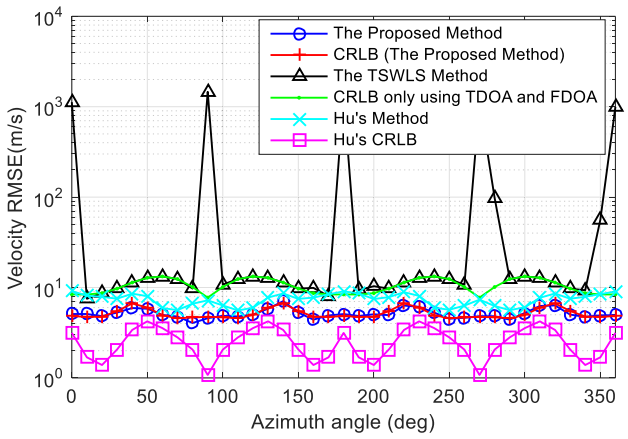

(f) RMSEs of velocity with radius of $1000 \mathrm{~m}$

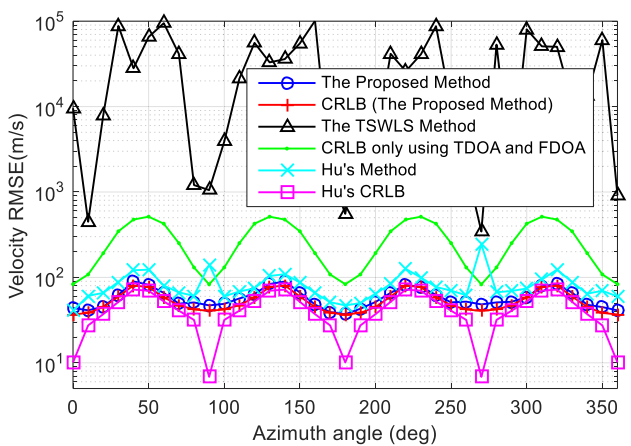

(h) RMSEs of velocity with radius of $4000 \mathrm{~m}$

Fig. 4 (See legend on next page.) 
(See figure on previous page.)

Fig. 4 Robustness comparison among different methods with different radius when $S N R=10 \mathrm{~dB}$ and $\sigma_{s}=10^{0.5} \mathrm{~m}$. a RMSEs of position with radius of $20 \mathrm{~m}$. b RMSEs of velocity with radius of $20 \mathrm{~m}$. c RMSEs of position with radius of $500 \mathrm{~m}$. d RMSEs of velocity with radius of $500 \mathrm{~m}$. e RMSEs of position with radius of $1000 \mathrm{~m}$. $\mathbf{f}$ RMSEs of velocity with radius of $1000 \mathrm{~m}$. $\mathbf{g}$ RMSEs of position with radius of $4000 \mathrm{~m}$. $\mathbf{h}$ RMSEs of velocity with radius of $4000 \mathrm{~m}$

always suffers the rank deficiency problem in some special points. Moreover, Hu's method cannot achieve its CRLB and is not suitable for scenarios with receiver location errors. On the contrary, the proposed method always attains its CRLB well and performs a better accuracy wherever the source moves, which exhibits strong robustness and better estimation performance.

\subsection{Performance comparison}

In order to further evaluate the efficiency, we study the localization performance of the proposed solution in terms of RMSE corresponding to different receiver location errors and SNRs. We compare its performance against Hu's method in [17], TSWLS method in [25], as well as the three types of CRLBs via Monte Carlo simulations. It should be explained that the improved TSWLS method in [14] is not simulated in this section, because this method used the TDOA and FDOA measurements only and not considered the receiver location errors, which will definitely result in some negative influences on estimation. The source location where $R=$ $500 \mathrm{~m}$ and $\phi=\pi / 3$ are set in this simulation, which is a robust position for the compared methods. SNR is equal to $10 \mathrm{~dB}$ when we investigate the estimation performance by varying receiver location error $\sigma_{s} . \sigma_{s}$ is set to $10^{0.5} \mathrm{~m}$ when SNR changes. Other simulation conditions are the same as that in Section 6.1.

Figure 5 shows the RMSEs comparison among different methods versus receiver location error when SNR = $10 \mathrm{~dB}$. The excess RMSE for the TSWLS method is of course due to the fact that it does not employ the differential Doppler rate measurements. Both Hu's method and the proposed method give a similar performance by attaining the CRLB at a sufficiently low level of the receiver location errors. Note that, in comparison with other methods, the proposed method attains the CRLB well as $\sigma_{s}$ increases especially for velocity estimate. Even though there is slightly above the CRLB for position estimate at $25 \mathrm{~dB}$, it is still much smaller than those using Hu's method, which deviates from the CRLB early at $0 \mathrm{~dB}$. Moreover, the superiority of the proposed method becomes more and more obvious with the increase of $\sigma_{s}$ because of considering the receiver location errors in the process of method solved.

Figure 6 provides the performance comparison of different methods as SNR changes from -20 to $30 \mathrm{~dB}$, and the level of receiver location error is fixed at $10^{0.5} \mathrm{~m}$. From Fig. 6, we conclude that the proposed solution still has the best performance and its RMSE curve always attains CRLB derived in Section 4.1 until SNR decreases to $-10 \mathrm{~dB}$. However, Hu's method cannot achieve the CRLB even at high SNR. The reason is that the first step of Hu's method could not provide a proper initial value for its second iteration step because of the existence of the receive location errors, which would easily converge to the local optimal. As for the proposed method, there is no convergence or initialization problem due to its algebraic solution expression. With the decrease of SNR, the performance deterioration phenomenon of Hu's method would become more and more obvious, but the proposed method still exhibits good performance. It proves again that the receiver location errors can limit

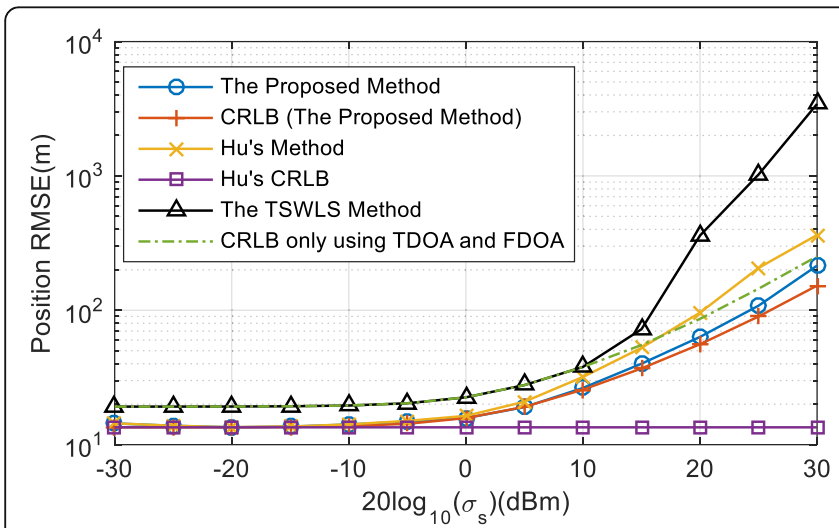

(a) RMSEs of position

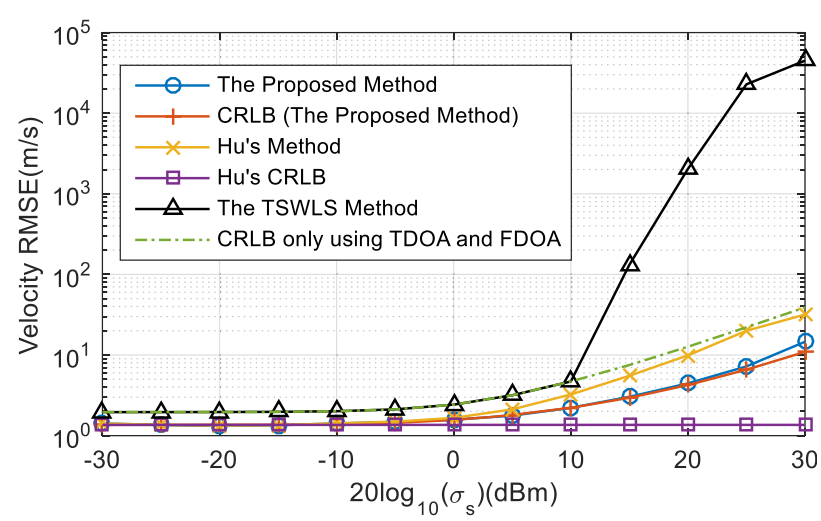

(b) RMSEs of velocity

Fig. 5 RMSEs comparison versus receiver location error when $S N R=10 \mathrm{~dB}$. a RMSEs of position. b RMSEs of velocity 


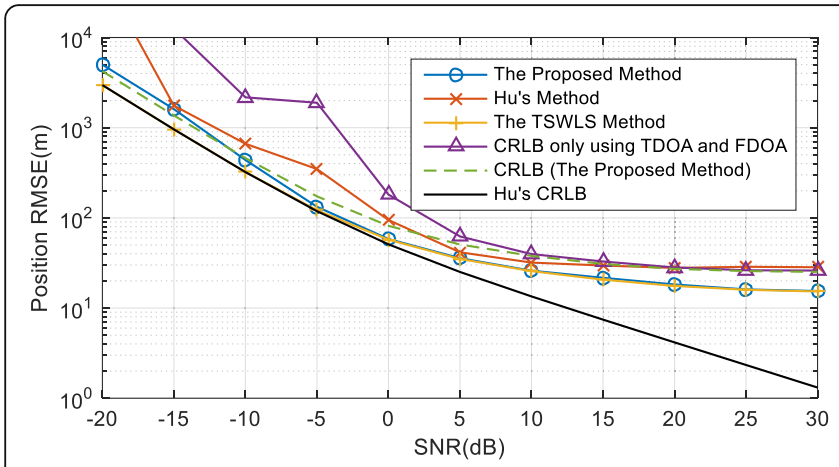

(a) RMSEs of position

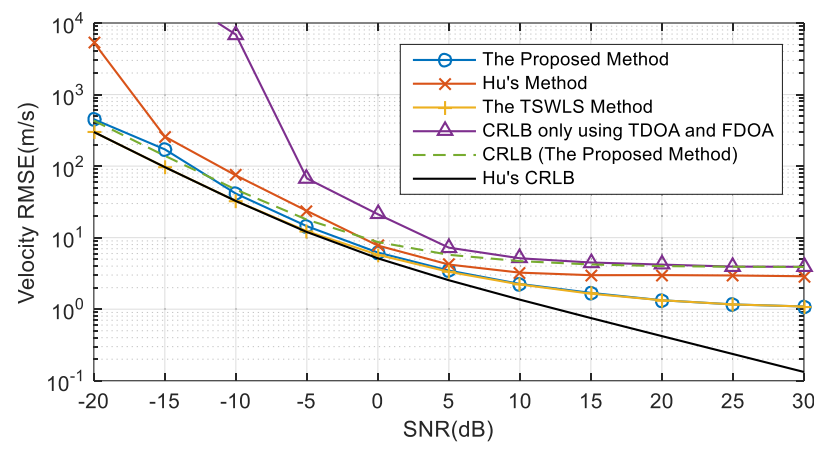

(b) RMSEs of velocity

Fig. 6 RMSE comparison versus SNR when $\sigma_{s}=10^{0.5} \mathrm{~m}$. a RMSEs of position. $\mathbf{b}$ RMSEs of velocity

the localization accuracy and the necessity of considering the receiver location errors in the method.

\subsection{Running time comparison}

The computational complexity is also another important index for evaluating the performance of the method. For this purpose, Table 2 compares the time cost of the proposed method, Hu's method [17], and the TSWLS method [25]. It should be pointed out that the running time is obtained from the average time of 1000 independent runs. The main configurations of the computer are listed as the following: Intel(R) Core(TM) i7-6700 CPU @ 3.40GHz; 8.00G RAM.

It can be seen from Table 2, Hu's method and the proposed method cost more time than the TSWLS methods because the additional differential Doppler rate measurement is added into the traditional TDOA-and-FDOA-based method. The running time of the proposed method is approximately three times larger than that of the Hu's method even using the same positioning measurements, which endures the relatively heavy computational burden among the methods. This is hardly surprising since the proposed method takes the receiver location errors into consideration while the existing methods did not. That is to say, it is at the expense of the higher computation cost that the proposed method achieves higher localization accuracy. In consideration of remarkable performance improvement, the increased but acceptable complexity is worthy.

Table 2 The comparison of the method in terms of average running time

\begin{tabular}{ll}
\hline Methods & $\begin{array}{l}\text { Running time } \\
\text { (ms) }\end{array}$ \\
\hline $\begin{array}{l}\text { The proposed method (using TDOA, FDOA, and } \\
\text { differential Doppler rate with receiver location errors) }\end{array}$ & 5.0714 \\
Hu's method [17] (using TDOA, FDOA, and differential & 1.5132 \\
$\begin{array}{l}\text { Doppler rate without receiver location errors) } \\
\text { The TSWLS method [25] (using TDOA and FDOA only) }\end{array}$ & 0.9123 \\
\hline
\end{tabular}

\section{Discussion}

To summarize, this paper mitigates the effects of receiver location errors in the localization accuracy and gives a final algebraic solution without extra mathematics operations usually used in existing methods. Based on the simulation results, we can observe that the proposed method can effectively avoid the rank deficiency problem when the source moves around. Moreover, the proposed method achieves the corresponding CRLB well at moderate noise and error levels and produces a remarkable gain in estimation accuracy compared with the existing methods for low SNRs and large receiver location errors. However, the complexity of the proposed method is higher than that of the existing methods because the proposed method considers the receiver location errors into the measurement model, while the existing methods did not. And this solution is for the localization of a single source only. We currently focus on extending it to the multiple source localization scenario with lower computational complexity. In addition, the estimation accuracy of TDOA, FDOA, and differential Doppler rate distinctly affect localization accuracy; hence, we intend to propose a high accuracy estimation method in our further study.

\section{Conclusions}

To improve localization accuracy and make the localization model closer to the practical environment, an algebraic method for moving source localization based on TDOA, FDOA, and differential Doppler rate measurements in the presence of receiver location errors is presented in this paper. The proposed method gives a final algebraic solution without iteration and extra mathematics operations by employing the basic idea of WLS processing, while the existing methods did not. The consideration of receiver location errors makes the proposed method achieve CRLB, no matter whether there exist receiver location errors or not. The proposed method was compared with several existing methods and showed 
generally better robustness and performance as SNR and receiver location error change both theoretically and numerically.

\section{Appendix 1}

\subsection{Detailed derivation of $\partial a^{\circ} / \partial_{2}^{\circ T}$}

The partial derivative $\partial \boldsymbol{\alpha}^{o} / \partial \boldsymbol{\theta}_{2}^{o T}$ can be written into the submatrice form as

$$
\frac{\partial \mathbf{\alpha}^{o}}{\partial \boldsymbol{\theta}_{2}^{o T}}=\left[\begin{array}{cc}
\frac{\partial \boldsymbol{r}^{o}}{\partial \boldsymbol{u}^{o T}} & \frac{\partial \boldsymbol{r}^{o}}{\partial \dot{\boldsymbol{u}}^{o T}} \\
\frac{\partial \dot{\boldsymbol{r}}^{o}}{\partial \boldsymbol{u}^{o T}} & \frac{\partial \dot{\boldsymbol{i}}^{o T}}{\partial \ddot{\boldsymbol{r}}^{o}} \\
\frac{\partial \dot{\boldsymbol{r}}^{o}}{\partial \boldsymbol{u}^{o T}} & \partial \dot{\boldsymbol{u}}^{o T}
\end{array}\right]_{3(M-1) \times 6}
$$

According to (4), (5), and (8), let

$$
\begin{aligned}
\boldsymbol{a}_{i}^{o} & =\frac{\left(\boldsymbol{u}^{o}-\boldsymbol{s}_{i}^{o}\right)^{T}}{r_{i}^{o}} \\
\boldsymbol{b}_{i}^{o} & =\frac{\left(\dot{\boldsymbol{u}}^{o}-\dot{\boldsymbol{s}}_{i}^{o}\right)^{T}}{r_{i}^{o}}-\frac{\dot{r}_{i}^{o}\left(\boldsymbol{u}^{o}-\boldsymbol{s}_{i}^{o}\right)^{T}}{r_{i}^{o 2}} \\
\boldsymbol{c}_{i}^{o} & =\frac{2 \dot{r}_{i}^{o 2}\left(\boldsymbol{u}^{o}-\boldsymbol{s}_{i}^{o}\right)^{T}}{r_{i}^{o 3}}-\frac{2 \dot{r}_{i}^{o}\left(\dot{\boldsymbol{u}}^{o}-\dot{\boldsymbol{s}}_{i}^{o}\right)^{T}}{r_{i}^{o 2}}-\frac{\ddot{r}_{i}^{o}\left(\boldsymbol{u}^{o}-\boldsymbol{s}_{i}^{o}\right)^{T}}{r_{i}^{o 2}}
\end{aligned}
$$

for $i=1,2, \ldots, M$. Thus, the partial derivatives of $r^{o}$ and $\dot{\boldsymbol{r}}^{o}$ with respect to $\boldsymbol{u}^{o}$ and $\dot{\boldsymbol{u}}^{o}$ yields (61), shown as

$$
\begin{gathered}
\frac{\partial \boldsymbol{r}^{o}}{\partial \boldsymbol{u}^{o T}}=\frac{\partial \dot{\boldsymbol{r}}^{o}}{\partial \dot{\boldsymbol{u}}^{T T}}=\left[\begin{array}{c}
\boldsymbol{a}_{2}^{o}-\boldsymbol{a}_{1}^{o} \\
\vdots \\
\boldsymbol{a}_{M}^{o}-\boldsymbol{a}_{1}^{o}
\end{array}\right]_{(M-1) \times 3}, \frac{\partial \dot{\boldsymbol{r}}^{o}}{\partial \boldsymbol{u}^{o T}}=\left[\begin{array}{c}
\boldsymbol{b}_{2}^{o}-\boldsymbol{b}_{1}^{o} \\
\vdots \\
\boldsymbol{b}_{M}^{o}-\boldsymbol{b}_{1}^{o}
\end{array}\right]_{(M-1) \times 3} \\
\frac{\partial \boldsymbol{r}^{o}}{\partial \dot{\boldsymbol{u}}^{o T}}=\boldsymbol{O}_{(M-1) \times 3}, \quad \frac{\partial \ddot{\boldsymbol{r}}^{o}}{\partial \dot{\boldsymbol{u}}^{o T}}=2 \frac{\partial \dot{\boldsymbol{r}}^{o}}{\partial \boldsymbol{u}^{o T}}, \quad \frac{\partial \ddot{\boldsymbol{r}}^{o}}{\partial \boldsymbol{u}^{o T}}=\left[\begin{array}{c}
\boldsymbol{c}_{2}^{o}-\boldsymbol{c}_{1}^{o} \\
\vdots \\
\boldsymbol{c}_{M}^{o}-\boldsymbol{c}_{1}^{o}
\end{array}\right]_{(M-1) \times 3}
\end{gathered}
$$

\section{Appendix 2}

\subsection{Detailed derivation of $\partial \alpha^{\circ} / \partial \beta^{\circ T}$}

In a similar manner, the partial derivative $\partial \boldsymbol{\alpha}^{o} / \partial \boldsymbol{\beta}^{o T}$ can be written as

$$
\frac{\partial \boldsymbol{\alpha}}{\partial \boldsymbol{\beta}^{o T}}=\left[\begin{array}{ll}
\frac{\partial \boldsymbol{r}^{o}}{\partial \boldsymbol{s}^{o T}} & \frac{\partial \boldsymbol{r}^{o}}{\partial \dot{\boldsymbol{s}}^{o T}} \\
\frac{\partial \dot{\boldsymbol{r}}^{o}}{\partial \dot{\boldsymbol{s}}^{o T}} & \frac{\partial \dot{\boldsymbol{r}}^{o T}}{\partial \dot{\boldsymbol{s}}^{o T}} \\
\frac{\partial \ddot{\boldsymbol{r}}^{o}}{\partial \dot{\boldsymbol{s}}^{o T}} & \frac{\dot{\boldsymbol{s}}^{o T}}{{ }^{o T}}
\end{array}\right]_{3(M-1) \times 6 M}
$$

with the elements of submatrices are given by

$$
\begin{aligned}
\frac{\partial \boldsymbol{r}^{o}}{\partial \boldsymbol{s}^{o T}} & =\left[\begin{array}{ccccc}
\boldsymbol{a}_{1}^{o} & -\boldsymbol{a}_{2}^{o} & & & \\
\boldsymbol{a}_{1}^{o} & & -\boldsymbol{a}_{3}^{o} & & \\
\vdots & & & \ddots & \\
\boldsymbol{a}_{1}^{o} & & & & -\boldsymbol{a}_{M}^{o}
\end{array}\right], \quad \frac{\partial \dot{\boldsymbol{r}}^{o}}{\partial \boldsymbol{s}^{o T}} \\
& =\left[\begin{array}{ccccc}
\boldsymbol{b}_{1}^{o} & -\boldsymbol{b}_{2}^{o} & & & \\
\boldsymbol{b}_{1}^{o} & & -\boldsymbol{b}_{3}^{o} & & \\
\vdots & & & \ddots & \\
\boldsymbol{b}_{1}^{o} & & & & -\boldsymbol{b}_{M}^{o}
\end{array}\right], \quad \frac{\partial \ddot{\boldsymbol{r}}^{o}}{\partial \boldsymbol{s}^{o T}} \\
& =\left[\begin{array}{ccccc}
\boldsymbol{c}_{1}^{o} & -\boldsymbol{c}_{2}^{o} & & & \\
\boldsymbol{c}_{1}^{o} & & -\boldsymbol{c}_{3}^{o} & & \\
\vdots & & & \ddots & \\
\boldsymbol{c}_{1}^{o} & & & & -\boldsymbol{c}_{M}^{o}
\end{array}\right]
\end{aligned}
$$

and

$$
\begin{aligned}
\frac{\partial \ddot{\boldsymbol{r}}^{o}}{\partial \dot{\boldsymbol{s}}^{o T}} & =2 \frac{\partial \dot{\boldsymbol{r}}^{o}}{\partial \boldsymbol{s}^{o T}}, \quad \frac{\partial \dot{\boldsymbol{r}}^{o}}{\partial \dot{\boldsymbol{s}}^{o T}}=\frac{\partial \boldsymbol{r}^{o}}{\partial \boldsymbol{s}^{o T}}, \quad \frac{\partial \boldsymbol{r}^{o}}{\partial \dot{\boldsymbol{s}}^{O T}} \\
& =\boldsymbol{O}_{(M-1) \times 3 M}
\end{aligned}
$$

for $i=1,2, \ldots, M$.

Abbreviations

3-D: Three-dimensional; AOA: Angle of arrival; CRLB: Cramér-Rao lower bound; FDOA: Frequency difference of arrival; RMSE: Root mean square error; RSS: Received signal strength; SNR: Signal-to-noise ratio; TDOA: Time difference of arrival; TOA: Time of arrival; TSWLS: Two-step weighted least square; WLS: Weighted least square

\section{Acknowledgements}

The authors would like to thank the Editorial board and anonymous Reviewers for their careful reading and constructive comments which provide an important guidance for our paper writing and research work. The authors would also like to thank K. C. Ho for their previous studies, which helped us very much.

\section{Funding}

This work was supported by the National Natural Science Foundation of China under Grant 61703433.

\section{Availability of data and materials}

The datasets generated during the current study are not publicly available but are available from the corresponding author on reasonable request.

\section{Authors' contributions}

$\mathrm{ZL}$ and $\mathrm{DH}$ derived the theoretical of the method and wrote the manuscript. $Y Z$ and JZ were in charge of the experiment and results. All authors had a significant contribution to the development of early ideas and design of the final methods. All authors read and approved the final manuscript.

Ethics approval and consent to participate

This paper does not contain any studies with human participants or animals performed by any of the authors.

\section{Consent for publication}

Informed consent was obtained from all authors included in the study.

\section{Competing interests}

The authors declare that they have no competing interests.

\section{Publisher's Note}

Springer Nature remains neutral with regard to jurisdictional claims in published maps and institutional affiliations. 
Received: 5 September 2018 Accepted: 27 March 2019

Published online: 11 April 2019

\section{References}

1. B. Lei, Y. Yang, K. Yang, Y. Wang, Y. Shi, A hybrid passive localization method under strong interference with a preliminary experimental demonstration. EURASIP J. Adv. Signal Process. 2016(1), 1-9 (2016). https://doi.org/10.1186/ s13634-016-0430-3

2. D. Wang, J. Yin, R. Liu, Z. Wu, T. Tang, Robust direct position determination methods in the presence of array model errors. EURASIP J. Adv. Signal Process. 2018(1), 1-28 (2018). https://doi.org/10.1186/s13634-018-0555-7

3. Z. Liu, Y. Zhao, D. Hu, C. Liu, A moving source localization method for distributed passive sensor using TDOA and FDOA measurements. Int. J. Antennas Propag. 2016(4), 1-12 (2016). https://doi.org/10.1155/2016/ 8625039

4. J. Li, F. Guo, L. Yang, W. Jiang, H. Pang, On the use of calibration sensors in source localization using TDOA and FDOA measurements. Digital Signal Process. 27(1), 33-43 (2014). https://doi.org/10.1016/j.dsp.2013.12.011

5. B. Beck, S. Lanh, R. Baxley, X. Ma, Uncooperative emitter localization using signal strength in uncalibrated mobile networks. IEEE Trans. Wirel. Commun. 16(11), 7488-7500 (2017). https://doi.org/10.1109/TWC.2017.2749231

6. E. Xu, Z. Ding, S. Dasgupta, Source localization in wireless sensor networks from signal time-of-arrival measurements. IEEE Trans. Signal Process. 59(6), 2887-2897 (2011). https://doi.org/10.1109/TSP.2011.2116012

7. J. Shen, A.F. Molisch, J. Salmi, Accurate passive location estimation using TOA measurements. IEEE Trans. Wirel. Commun. 11(6), 2182-2192 (2012). https://doi.org/10.1109/TWC.2012.040412.110697

8. X. Ouyang, Q. Wan, J. Cao, J. Xiong, Q. He, Direct TDOA geolocation of multiple frequency-hopping emitters in flat fading channels. IET. Signal Process. 11(1), 80-85 (2017). https://doi.org/10.1049/iet-spr.2016.0299

9. N.O. TK Le, Closed-form and near closed-form solutions for TDOA-based joint source and sensor localization. IEEE Trans. Signal Process. 64(18), 47514766 (2016). https://doi.org/10.1109/TSP.2016.2633784

10. K.C. Ho, W. Xu, An accurate algebraic solution for moving source location using TDOA and FDOA measurements. IEEE Trans. Signal Process. 52(9), 2453-2463 (2004). https://doi.org/10.1109/TSP.2004.831921

11. W.H. Foy, Position-location solutions by Taylor-series estimation. IEEE Trans, Aerosp. Electron. Syst. AES 12(2), 187-194 (2007). https://doi.org/10.1109/ TAES.1976.308294

12. G. Wang, Y. Li, N. Ansari, A semidefinite relaxation method for source localization using TDOA and FDOA measurements. IEEE Trans. Veh. Technol. 62(2), 853-862 (2013). https://doi.org/10.1109/TVT.2012.2225074

13. Y. Wang, Y. Wu, An efficient semidefinite relaxation algorithm for moving source localization using TDOA and FDOA measurements. IEEE Commun. Lett. 21(1), 80-83 (2017). https://doi.org/10.1109/LCOMM.2016.2614936

14. A. Noroozi, A.H. Oveis, M.R. Hosseini, M.A. Sebt, Improved algebraic solution for source localization from TDOA and FDOA measurements. IEEE Wireless Commun. Lett. 7(3), 352-355 (2018). https://doi.org/10.1109/LWC.2017. 2777995

15. X. Chen, J. Guan, N. Liu, Y. He, Maneuvering target detection via radonfractional Fourier transform-based long-time coherent integration. IEEE Trans. Signal Process. 62(4), 939-953 (2014). https://doi.org/10.1109/TSP. 2013.2297682

16. X. Li, G. Cui, W. Yi, L. Kong, Coherent integration for maneuvering target detection based on Radon-Lv's distribution. IEEE Signal Process. Lett 22(9), 1467-1471 (2015). https://doi.org/10.1109/LSP.2015.2390777

17. H. D, Z. Huang, X. Chen, L. J, A moving source localization method using TDOA, FDOA and Doppler rate measurements. IEICE Trans. Commun. E99B(3), 758-766 (2016). https://doi.org/10.1587/transcom.2015EBP3355

18. S Tao, T Ran, SR Rong, A fast method for time delay, Doppler shift and Doppler rate estimation. Paper presented at IEEE International Conference on Radar, Guangzhou, China, 2016.

19. D. Hu, Z. Huang, S. Zhang, J. Lu, Joint TDOA, FDOA and differential Doppler rate estimation: method and its performance analysis. Chin. J. Aeronaut. 31(1), 137-147 (2018). https://doi.org/10.1016/j.cja.2017.11.005

20. X. Xiao, F. Guo, Joint estimation of time delay, Doppler velocity and Doppler rate of unknown wideband signals. Circ. Syst. Signal Process. 2018(8), 1-20 (2018). https://doi.org/10.1007/s00034-018-0816-6

21. P. Bello, Joint estimation of delay, Doppler, and Doppler rate. IRE Trans. Inf. Theory 6(3), 330-341 (2003). https://doi.org/10.1109/TIT.1960.1057562
22. Q. Lin, T. Ran, S. Zhou, Y. Wang, Detection and parameter estimation of multicomponent LFM signal based on the fractional Fourier transform. Sci. China Inform. Sci. 47(2), 184-198 (2004). https://doi.org/10.1360/02yf0456

23. L. Rui, K.C. Ho, Elliptic localization: performance study and optimum receiver placement. IEEE Trans. Signal Process. 62(18), 4673-4688 (2014). https://doi. org/10.1109/TSP.2014.2338835

24. B.K. Chalise, Y.D. Zhang, M.G. Amin, B. Himed, Target localization in a multistatic passive radar system through convex optimization. Signal Process. 102, 207-215 (2014). https://doi.org/10.1016/j.sigpro.2014.02.023

25. K.C. Ho, X. Lu, L. Kovavisaruch, Source localization using TDOA and FDOA measurements in the presence of receiver location errors: analysis and solution. IEEE Trans. Signal Process. 55(2), 684-696 (2007). https://doi.org/10. 1109/TSP.2006.885744

26. KB Petersen, MS Pedersen, The Matrix Cookbook. Available: http://www2. imm.dtu.dk/pubdb/p.php?3274 (2012)

27. W. Ding, B. Tan, Y. Chen, F.N. Teferle, Y. Yuan, Evaluation of a regional realtime precise positioning system based on GPS/BeiDou observations in Australia. Adv. Space Res. 61(3), 951-961 (2008). https://doi.org/10.1016/j.asr. 2017.11.009

28. S. Bian, J. Jin, Z. Fang, The Beidou satellite positioning system and its positioning accuracy. Navigation 52(3), 123-129 (2005). https://doi.org/10. 1002/j.2161-4296.2005.tb01739.x

\section{Submit your manuscript to a SpringerOpen ${ }^{\circ}$ journal and benefit from:}

- Convenient online submission

- Rigorous peer review

- Open access: articles freely available online

High visibility within the field

- Retaining the copyright to your article

Submit your next manuscript at $>$ springeropen.com 